ARTICLE

\title{
Arabidopsis P4 ATPase-mediated cell detoxification confers resistance to Fusarium graminearum and Verticillium dahliae
}

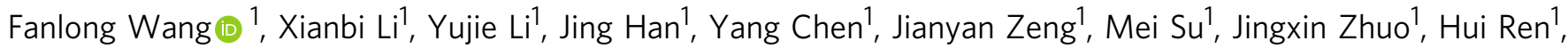
Haoru Liu', Lei Hou', Yanhua Fan ${ }^{1}$, Xingying Yan¹, Shuiqing Song ${ }^{1}$, Juan Zhao ${ }^{1}$, Dan Jin ${ }^{1}$, Mi Zhang (1) ${ }^{1} \&$ Yan Pei (1) ${ }^{1 凶}$

Many toxic secondary metabolites produced by phytopathogens can subvert host immunity, and some of them are recognized as pathogenicity factors. Fusarium head blight and Verticillium wilt are destructive plant diseases worldwide. Using toxins produced by the causal fungi Fusarium graminearum and Verticillium dahliae as screening agents, here we show that the Arabidopsis P4 ATPases AtALA1 and AtALA7 are responsible for cellular detoxification of mycotoxins. Through AtALA1-/AtALA7-mediated vesicle transport, toxins are sequestered in vacuoles for degradation. Overexpression of AtALA1 and AtALA7 significantly increases the resistance of transgenic plants to F. graminearum and V. dahliae, respectively. Notably, the concentration of deoxynivalenol, a mycotoxin harmful to the health of humans and animals, was decreased in transgenic Arabidopsis siliques and maize seeds. This vesicle-mediated cell detoxification process provides a strategy to increase plant resistance against different toxinassociated diseases and to reduce the mycotoxin contamination in food and feed.

\footnotetext{
${ }^{1}$ The Affiliation Biotechnology Research Center, Southwest University, No. 2 Tiansheng Rd, Beibei, Chongqing 400716, P. R. China.

$凶_{\text {email: peiyan3@swu.edu.cn }}$
} 
P lant fungal diseases cause serious production and economic losses in agriculture and forestry worldwide. Many important phytopathogenic fungi, such as Fusarium species and Verticillium species, produce secondary metabolites that are toxic to host cells ${ }^{1-3}$. F. graminearum is the causal agent of Fusarium head blight (FHB) disease in cereals, which results in significant yield losses mainly in wheat, barley, and maize ${ }^{4-11}$. During pathogenic processes, the fungus produces epoxy-sesquiterpenoid compounds, named trichothecenes, including deoxynivalenol (DON), T-2 toxin, HT-2 toxin, and nivalenol ${ }^{7,12}$. These mycotoxins can evoke the rapid generation of reactive oxygen species that stimulate apoptosis-like processes and result in chlorotic and necrotic lesions, growth cessation, or even plant death ${ }^{13-17}$. Owing to their ability to inhibit protein synthesis, trichothecenes are also toxic to mammals. In fact, the contamination of trichothecene mycotoxins in food and feed commodities has become a global safety issue ${ }^{18-20}$. Verticillium wilt, mainly caused by the soil-borne vascular fungi $V$. dahliae and V. albo-atrum, is another destructive plant disease that results in tremendous economic losses worldwide. These diseases can be found in over 200 plant species, including many annual crops and perennial trees $^{21-31}$. Owing to the complicated pathogenic mechanisms and the lack of host resistance to F. graminearum, successful control of FHB has been a major challenge for breeders ${ }^{32,33}$. Likewise, with a broad host range, high genetic diversity, and long-term persistence in the soil, Verticillium species have been ranked as some of the most difficult pathogens to control ${ }^{27,30,34}$

Mycotoxins have been regarded as the key virulence factors of FHB and Verticillium wilt ${ }^{24,35}$. Therefore, the detoxification of mycotoxins has been developed to control these formidable diseases. This strategy protects hosts from the toxicity of mycotoxins, thus allowing plants to maintain their innate immunity against the invasion of pathogens and increase their resistance to diseases caused by toxin producers.

In living cells, there are three major strategies for detoxification: (i) chemical modification/inactivation, (ii) compartmentation, and (iii) efflux ${ }^{18}$. At present, the main strategy used to detoxify trichothecenes in plant breeding is chemical modification/inactivation. UDP-glucosyltransferase (UGT) can conjugate DON into nontoxic DON-3-O-glucoside (D3G). DON resistance was achieved in Arabidopsis ${ }^{36}$ and wheat ${ }^{37}$ by overexpressing the UGT gene. In wheat, Fhb1 was identified to contribute to resistance to a broad spectrum of Fusarium species ${ }^{8,38-40}$. Recently, it was found that Fhb7 encodes a glutathione-S-transferase (GST) that could detoxify trichothecenes through de-epoxidation and thus confer broad resistance to Fusarium species ${ }^{8}$. However, the side effects on plant growth resulting from metabolic modification $^{36,41}$ and the toxicity reversal of the modified compounds $^{42,43}$ caused by this strategy remain to be solved.

Verticillium toxins include the high molecular weight protein lipopolysaccharide complex (PLPC) ${ }^{44-46}$, small peptides, and low molecular weight metabolites $24,47,48$. Among them, low molecular weight metabolites are the key causal agents of wilt symptoms $24,48,49$. A lipophilic phytotoxin named cinnamate acetate (CIA) was identified from a strain of $V$. dahliae that is pathogenic to olive trees; this compound can induce Verticillium wilt-like symptoms and thus could be used to select Verticilliumtolerant olive trees ${ }^{50}$. Although Verticillium toxins have been used for the selection of disease-resistant plants ${ }^{50,51}$, the targeted detoxification of these toxins has not been reported in plants due to the complicated composition of Verticillium toxins.

The P4 subfamily of P-type ATPases (P4 ATPases) functions as lipid flippases that translocate specific lipid substrates from the exoplasmic/luminal leaflet to the cytoplasmic leaflet of biological membranes, and these ATP-fueled flippases have a key role in the biogenesis of intracellular mobile vesicles ${ }^{52-62}$. Accumulating evidence in yeast, worms, plants and mammals has shown that P4 ATPase-mediated transport is involved in a variety of developmental and physiological processes, including the cell cycle, organelle biogenesis, embryogenesis, cellular polarization, hormone transport, and the response to biotic/abiotic stress ${ }^{63-66}$. In Arabidopsis, there are 12 members (AtALA1 to AtALA12; Arabidopsis thaliana aminophospholipid ATPases) in the P4 ATPase family. Although important roles of $\overline{A t} A L A s$ have been reported in tolerance to temperature stresses ${ }^{67-69}$, disease resistance ${ }^{70-72}$, auxin polar distribution ${ }^{73}$, cell expansion and plant growth ${ }^{74,75}$, pollen and ovule development ${ }^{76-78}$, little is known about the role of P4 ATPases in mycotoxin detoxification, even though ALA4 was demonstrated to be involved in heavy mental detoxification $^{79}$.

In this study, using the Fusarium toxin DON and Verticillium toxin CIA as probes, we identified two Arabidopsis P4 ATPase genes, AtALA1 and AtALA7 that were responsible for resistance to the toxins. We revealed that detoxification occurred via vesiclemediated transport of toxins from the plasma membrane to vacuoles. Importantly, the expression of AtALA7 in Arabidopsis and tobacco significantly increased the resistance to different strains of $V$. dahliae, whereas the exogenous overexpression of AtALA1 in maize significantly increased the resistance to DON and ear rot disease, thus largely reducing the DON concentration in seeds. Our data demonstrated that this vesicle transportmediated cell detoxification can be achieved without a growth penalty in different plant species targeting different toxins. Therefore, this strategy is promising for the development of resistance to recalcitrant diseases, e.g., FHB and Verticillium wilt, as well as for the reduction of mycotoxin contamination in food and feed.

\section{Results}

AtALA1 and AtALA7 are responsible for the vacuole compartmentation of DON and CIA. After treatment of AtALA1 to 11 loss-of-function Arabidopsis mutants (Supplementary Fig. 1) with DON $(50 \mu \mathrm{g} / \mathrm{mL})$ and CIA $(100 \mu \mathrm{g} / \mathrm{mL})$, mutants for AtALA1 (ala1, ala1-8) and AtALA7 (ala7, ala7-24) showed significantly reduced tolerance to DON (Fig. 1a-c and Supplementary Fig. 2a, b) and CIA (Fig. 1f-h and Supplementary Fig. 2e, f), respectively. To investigate whether defects in AtALA1 and AtALA7 could affect the accumulation of toxins in vacuoles, we treated ala1 and ala7 mutants with DON labeled with 5-carboxyfluorescein (DON $\left.{ }^{5-F A M}\right)$ and CIA labeled with fluorescein-isothiocyanate-isomer (CIA $\left.{ }^{\text {FITC}}\right)$, which did not impair the toxicity of either DON (Supplementary Fig. 3a, b) or CIA (Supplementary Fig. 3c, d). With DON ${ }^{5-F A M}$ treatment, no obvious fluorescent signal was observed in the vacuoles of cells of ala1 mutant, whereas the signal appeared obvious in the vacuoles of other mutants (Fig. 1d, e and Supplementary Fig. 2c, d). Similarly, the CIA ${ }^{\text {FITC }}$ signal was nearly undetectable in the ala vacuoles, whereas the signal appeared obvious in the vacuoles of other mutants (Fig. 1i, j and Supplementary Fig. 2g, h). Moreover, the dynamic trafficking of DON ${ }^{5-F A M}$ and CIA ${ }^{\text {FITC }}$ in wild-type Arabidopsis and the mutants was observed. At $2 \mathrm{~h}$, no

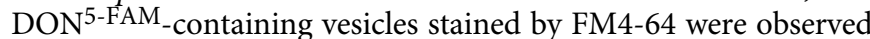
in root hair cells of the ala1 mutant. However, the DON ${ }^{5-F A M}$ signal was observed in the vesicles in root hair cells of wild-type and complemented plants (proAtALA1::EGFP-AtALA1, Comp1) (Fig. 1d). At $6 \mathrm{~h}$, the DON ${ }^{5-\mathrm{FAM}}$ signal could be observed in small vacuoles of root meristem cells of wild-type and complemented plants; at $10 \mathrm{~h}$, the signal accumulated in the mature vacuoles (Fig. 1d, e). In contrast, DON ${ }^{5-F A M}$ appeared in the cytoplasm of the ala1 mutant but not in vesicles or vacuoles (Fig. 1d). Similarly, 

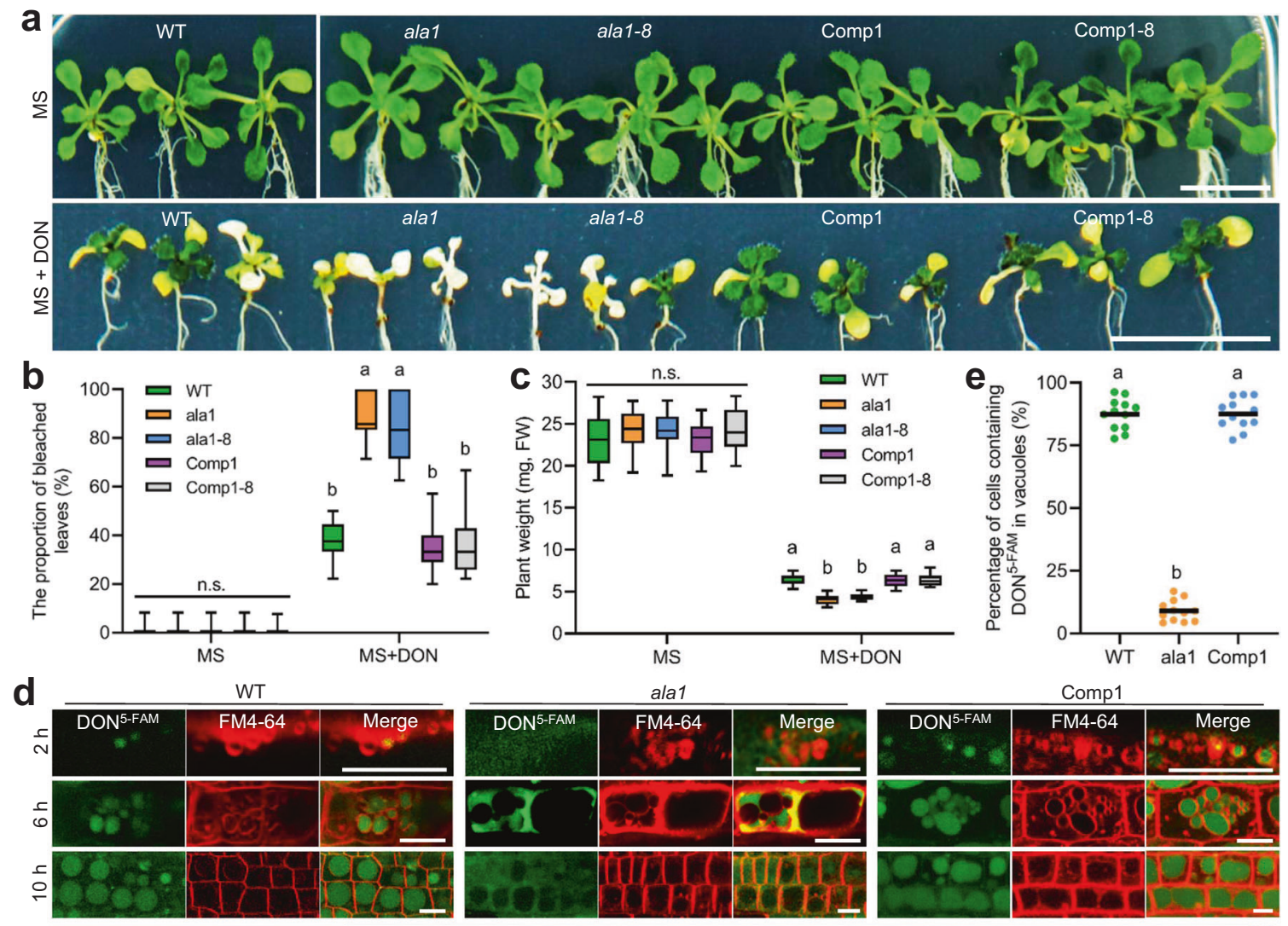

f
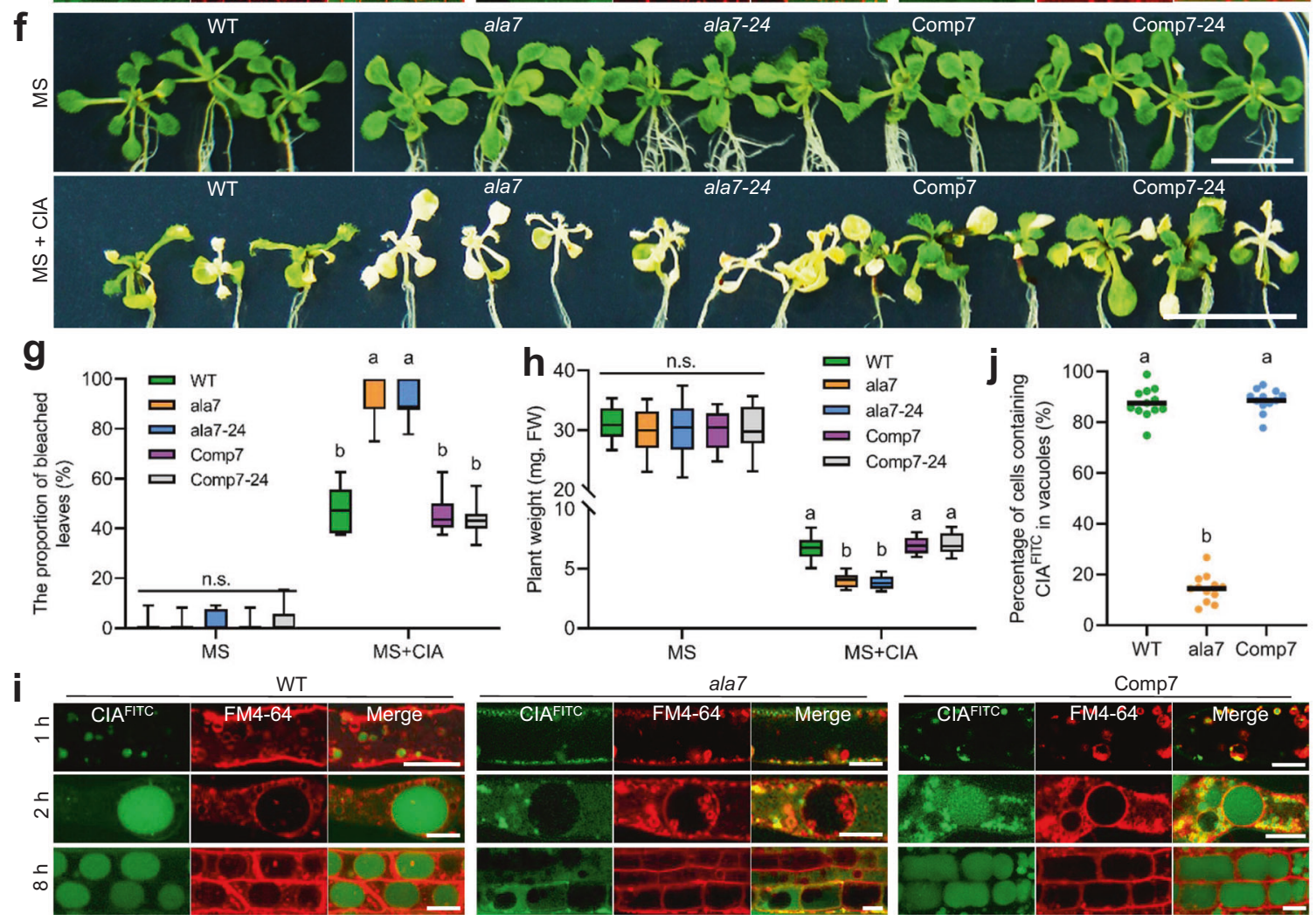

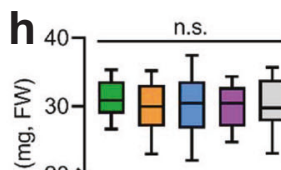
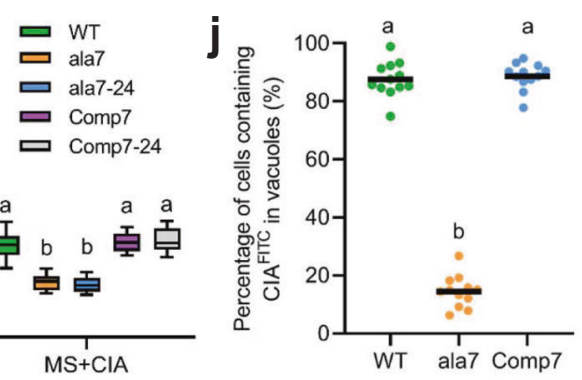

ala7
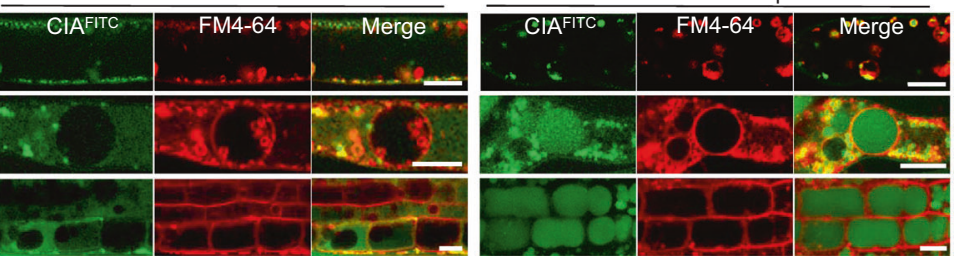

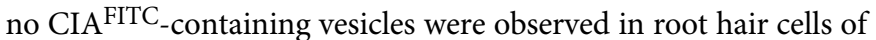
the ala 7 mutant at $1 \mathrm{~h}$ and $2 \mathrm{~h}$, whereas the signal clearly appeared in the vesicles at $2 \mathrm{~h}$ and accumulated in vacuoles of wild-type and complemented plants (proAtALA7::AtALA7-eGFP, Comp7) at $8 \mathrm{~h}$ (Fig. 1i, j). The decreased tolerance and defective accumulation in vacuoles of DON/CIA were rescued by the expression of AtALA1 and AtALA7 in ala1 and ala7 plants, respectively (Fig. 1). These data reveal that in Arabidopsis, AtALA1 is responsible for the vacuole accumulation of DON and that AtALA7 is responsible for the vacuole accumulation of CIA. 
Fig. 1 AtALA1 is responsible for the vacuolar compartmentation of DON, and AtALA7 is responsible for that of CIA. a DON tolerance assay of wildtype, and AtALA1 mutant (ala1, ala1-8) and complemented (Comp1, Comp1-8) Arabidopsis seedlings. The native promoter-controlled AtALA1 was used to rescue the defect of AtALA1 in mutants. Six-day-old seedlings were transferred to MS media containing DON ( $50 \mu \mathrm{g} / \mathrm{mL})$ for $7 \mathrm{~d}$. WT, wild-type; ala1 and ala1-8, AtALA1 loss-of-function mutant. Comp1 and Comp1-8, complemented ala1 and ala1-8 seedlings expressing proAtALA1::EGFP-AtALA1. DON, deoxynivalenol; proAtALA1, the native promoter of the AtALA1 gene. Scale bar, $1 \mathrm{~cm}$. b Proportion of bleached leaves suffering from DON. Data are presented as box-and-whisker plots of three replicates (eight plants each). c Weight of plants with or without DON treatment. Data are presented as boxand-whisker plots of three replicates (six plants each). $\mathbf{d}$ Distribution of DON5-FAM in root cells of wild-type, ala1 mutant, and complemented Arabidopsis seedlings. Seedlings were treated with DON ${ }^{5-F A M}(9 \mu \mathrm{g} / \mathrm{mL})$ and FM4-64 $(8 \mu \mathrm{M})$ for $2 \mathrm{~h}, 6 \mathrm{~h}$, and $10 \mathrm{~h}$. Confocal images at $2 \mathrm{~h}$ were collected from root hair cells, whereas those at $6 \mathrm{~h}$ and $10 \mathrm{~h}$ were collected from cells of the root meristem zone. At least three independent repeats were performed. DON5-FAM, DON labeled with 5-carboxyfluorescein (5-FAM). Scale bar, $10 \mu \mathrm{m}$. e Percentage of cells containing DON5-FAM in their vacuoles at 10 h. Data are presented as dot plots ( $n=12$ roots). $\mathbf{f}$ CIA tolerance assay of wild-type, AtALA7 mutant (ala7, ala7-24) and complemented (Comp7, Comp7-24) Arabidopsis seedlings. The native promoter-controlled AtALA7 was used to rescue the defect of AtALA7 in mutants. Six-day-old seedlings were transferred to MS media containing CIA $(100 \mu \mathrm{g} / \mathrm{mL})$ for 7 d. ala7 and ala7-24, AtALA7 loss-of-function mutant. Comp7 and Comp7-24, complemented ala7 and ala724 mutants seedlings expressing proAtALA7::AtALA7-eGFP. CIA, cinnamate acetate; proAtALA7, the native promoter of the AtALA7 gene. Scale bar, $1 \mathrm{~cm}$. $\mathbf{g}$ Proportion of bleached leaves resulting from CIA treatment. Data are shown as box-and-whisker plots of three replicates (eight plants each). $\mathbf{h}$ Weight of plants with or without CIA treatment. Data are presented as box-and-whisker plots of three replicates (six plants each). i Distribution of CIA ${ }^{\mathrm{FITC}}$ in root cells of wild-type, ala7 mutant and complemented (Comp7) Arabidopsis seedlings. The seedlings were treated with $\mathrm{CIA}$ FITC $(5 \mu \mathrm{g} / \mathrm{mL})$ and FM4-64 ( $8 \mu \mathrm{M})$ for $1 \mathrm{~h}, 2 \mathrm{~h}$, and $8 \mathrm{~h}$. Confocal images at $1 \mathrm{~h}$ and $2 \mathrm{~h}$ were collected from the root hair cells, while those at $10 \mathrm{~h}$ were collected from cells of the root meristem zone. At least three independent repeats were conducted. CIAFITC, CIA labeled with fluorescein-isothiocyanate-isomer (FITC). Scale bar, $0.5 \mu \mathrm{m}$.

j Percentage of cells containing CIAFITC in their vacuoles at $8 \mathrm{~h}$. Data are presented as dot plots ( $n=12$ roots). Box-and-whisker plots show the medians (horizontal lines), upper and lower quartiles (box edges), and $1.5 \times$ the interquartile range (whiskers). Different letters in $\mathbf{b}, \mathbf{c}, \mathbf{e}, \mathbf{g}, \mathbf{h}$, and $\mathbf{j}$ represent significant differences at $P<0.05$ by one-way ANOVA with Tukey multiple comparisons test. n.s., not significant.

DON/CIA are transported to vacuoles via AtALA1-/AtALA7mediated vesicle transport. To investigate the role of AtALA1 in DON transport, we generated transgenic 35S::RFP-AtALA1 and proAtALA1::EGFP-AtALA1 Arabidopsis. The DON ${ }^{5-F A M}$ signal was colocalized with RFP-AtALA1 in the plasma membrane, vesicles and vacuoles of root hairs (Fig. 2a), showing that the accumulation of DON in vacuoles is associated with the vesiclemediated transport pathway. To further trace the trafficking of DON in the cell, DON ${ }^{5-F A M}$ treatment was performed separately in two transgenic Arabidopsis lines expressing RFP-fused RabA1d, an early endosome (EE) marker ${ }^{80}$, and $\gamma$-Tip-RFP, a lytic vacuole marker ${ }^{81}$. The $\mathrm{DON}^{5-\mathrm{FAM}}$ signal appeared in RFPRabA1d-indicated EE-like structures (Fig. 2b); the signal converged in vacuoles that were surrounded by $\gamma$-Tip-RFP (Fig. 2c). The subcellular localization of AtALA1 was observed in proAtALA1::EGFP-AtALA1 transgenic Arabidopsis. In root hair cells, the signal was observed at the PM and tonoplast (Fig. 2d). The transient expression of AtALA1 in tobacco confirmed its localization at the PM (Supplementary Fig. $4 \mathrm{a}, \mathrm{b}$ ), which required the presence of ALIS $1^{82}$. The colocalization analysis of EGFPAtALA1 with the EE marker RFP-RabA1d and the late endosome (LE) marker RFP-RabF $2 a^{83}$ showed that EGFP-AtALA1 was colocalized with the two marker proteins (Fig. 2e, f). These data demonstrated that AtALA1 was localized to the PM, EE, LE and tonoplasts.

To verify that the vacuole accumulation of $\mathrm{DON}^{5-\mathrm{FAM}}$ is associated with vesicle-mediated transport, wild-type seedlings were treated with the vesicle trafficking inhibitors tyrphostin A23 (TyrA23), brefeldin A (BFA), and wortmannin (Wm). TyrA23 is a well-characterized inhibitor of the clathrin-mediated endocytic pathway, which can specifically block the interaction between the tyrosine motif of the cargo and the adaptor protein AP- $2^{84,85}$. Exposure of seedlings to TyrA23 led to a diffuse distribution of $\mathrm{DON}^{5-F A M}$ in the cytoplasm rather than accumulation in mature vacuoles (Fig. 2g), indicating that endocytosis of DON may be clathrin-dependent. BFA is another vesicle trafficking inhibitor that affects the Golgi complex and Golgi retrograde transport, which usually results in the formation of the BFA compartment (BFA body) in plants 86,87 . In the BFA- and DON ${ }^{5-F A M}$ - treated cells, massive DON ${ }^{5-F A M}$, and BFA-induced FM4-64 aggregates formed, but the DON ${ }^{5-F A M}$ signal was not colocalized to the BFA bodies (Fig. 2g), confirming that the pathway for DON entering vacuoles is independent of the Golgi. Wm is a PI3 kinase-specific inhibitor, and this compound is widely used to observe vesicular trafficking routes in cells, including endocytosis and vesicle transport $^{88,89}$. In $\mathrm{Wm}$-treated cells, the $\mathrm{DON}^{5-F A M}$ signal was diffusively distributed in the cytosol, and DON did not accumulate in vacuoles (Fig. 2g). Inhibition experiments and colocalization showed that DON was endocytosed from the PM and then transported through the EE and LE into lytic vacuoles, and this vesicle-associated transport might be mediated by AtALA1.

Similar approaches were used to detect the location and trafficking of AtALA7 and CIA in cells. The CIA FITC signal colocalized with AtALA7-RFP in vesicles in the root hairs of 35S::AtALA1-RFP transgenic Arabidopsis (Fig. 3a). The endocytosis of CIA FITC in the cells of the root meristem region was slower than that in root hair cells (Fig. 1i). In the root meristem region, at $180 \mathrm{~min}$ and $183 \mathrm{~min}$ after exposure, CIA ${ }^{\text {FITC }}$ fluorescent bubbles protruded from the PM; at $201 \mathrm{~min}$, CIA ${ }^{\text {FITC }}$ gathered together in the cytosol and formed prevacuolar compartment (PVC) structures (Fig. 3b); 6 hours later, the signal converged in lytic vacuoles (Fig. 3c). Inhibition assays (Fig. 3d) showed that the trafficking of CIA to the vacuole was also associated with vesicle-mediated transport. Subcellular localization results revealed that AtALA7-eGFP signals, similar to those of AtALA1, appeared in the PM (Fig. 3e), EE (Fig. 3f), LE (Fig. 3g), and tonoplasts (Fig. 3e). Transient expression of AtALA7-eGFP and ALIS1 in tobacco showed that similar to AtALA1, AtALA7 needs its $\beta$-subunit to leave the ER (Supplementary Fig. 4c, d). These data indicate a similar endocytosis pathway to transport CIA from the PM through the EE and LE into vacuoles.

Overexpression of AtALA7 promotes the accumulation of CIA in vacuoles and enhances resistance to Verticillium wilt disease. To test the role of AtALA7 in the tolerance of CIA, the gene was overexpressed under the control of the cauliflower mosaic virus 35 S promoter (35S::AtALA7) in Arabidopsis. Two transgenic lines (OE-9 and OE-12) with high transcription levels of AtALA7 (Supplementary Fig. 5b) were used for the bioassay of CIA resistance. Neither upregulation nor disruption of AtALA7 


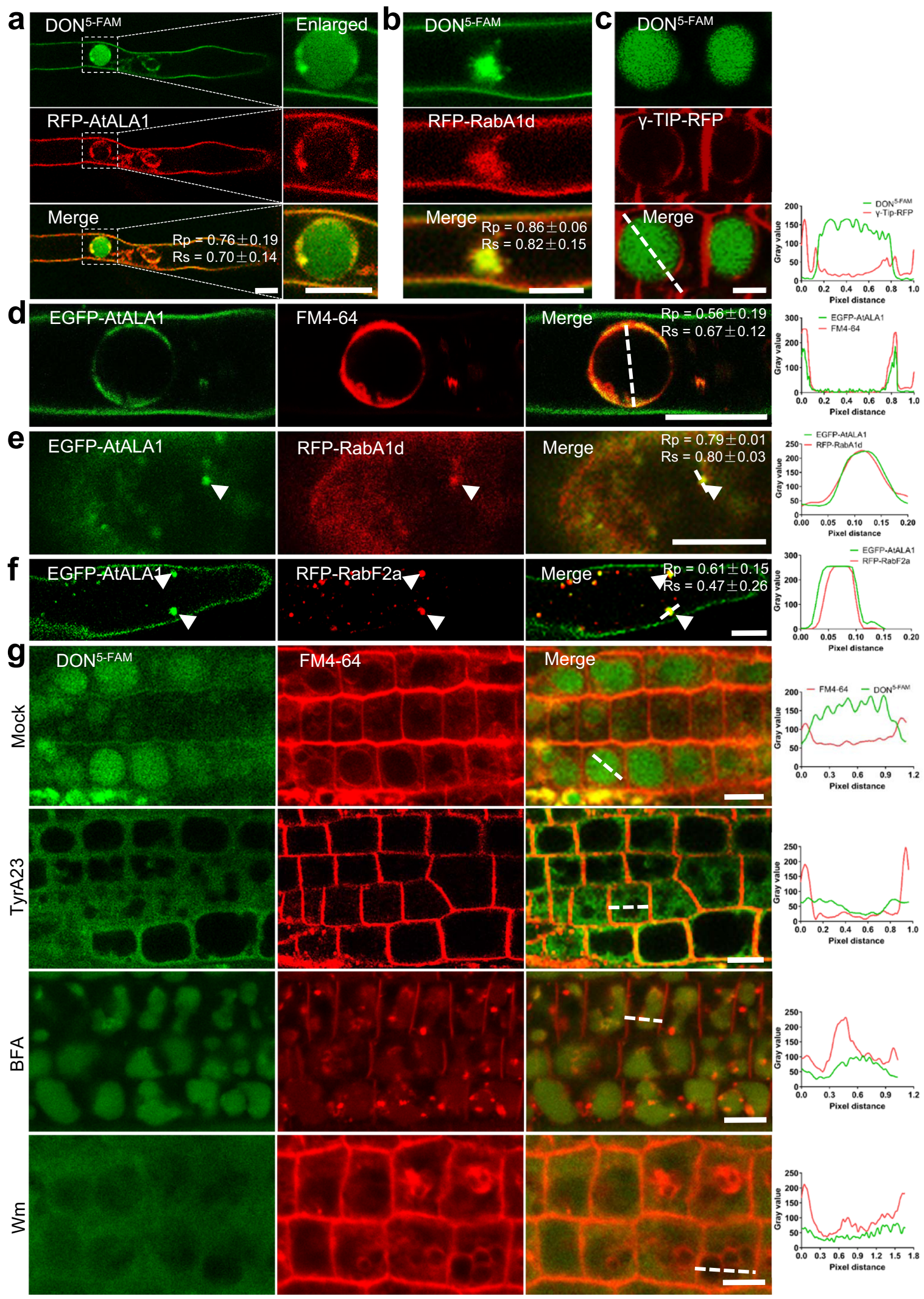

affected the growth of transgenic plants (Fig. 4a, b, Supplementary Fig. 1c and 5a, c, d). In the presence of CIA, wild-type plants showed toxic symptoms, including wilted leaves and decreased root growth. The symptoms were much more severe in the ala7 and ala7-24 mutants. In contrast, AtALA7-overexpressing lines (OE-9 and OE-12) showed less-severe disease than the wild-type plants (Fig. 4a-c). We then observed the distribution of CIA ${ }^{\text {FITC }}$ $(5 \mu \mathrm{g} / \mathrm{mL})$ in root cells and found that the CIA ${ }^{\text {FITC }}$ signal in vacuoles of $35 S::$ AtALA7 lines was much stronger than that of wild-type (Fig. 4d, e). To confirm this result, we generated 35S::AtALA7 tobacco and challenged two representative lines (Supplementary Fig. 6a) with CIA. The overexpression of AtALA7 
Fig. 2 DON5-FAM is transported via the EE and LE into lytic vacuoles. a DON5-FAM colocalized with RFP-AtALA1 in vesicles and EE-like structures in root hairs. In the vacuole of the root hair, DON ${ }^{5-F A M}$ colocalized with RFP-AtALA1 mainly in the tonoplasts. 35S::RFP-AtALA1 seedlings were treated with

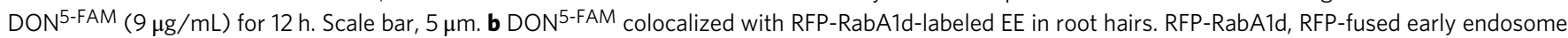
marker RabA1d. Six-day-old transgenic 35S::RFP-RabA1d seedlings were treated with DON5-FAM ( $9 \mu \mathrm{g} / \mathrm{mL})$ for $12 \mathrm{~h}$. Scale bar, $5 \mu \mathrm{m}$. c DON5-FAM was transported into $\gamma$-Tip-RFP labeled lytic vacuoles in the cells at the root meristem region. Six-day-old $35 S$ :: $\gamma$-Tip-RFP transgenic seedlings were treated with DON5-FAM $(9 \mu \mathrm{g} / \mathrm{mL})$ for $12 \mathrm{~h}$. Scale bar, $5 \mu \mathrm{m}$. d In root hair cells of proAtALA1::EGFP-AtALA1 transgenic Arabidopsis, EGFP-AtALA1 was localized to the PM and tonoplasts, as indicated by FM4-64 $(8 \mu \mathrm{M})$ staining. Scale bar, $10 \mu \mathrm{m}$. e In root hair cells of transgenic Arabidopsis containing proAtALA1::EGFP-AtALA1 and 35S::RFP-RabA1d, EGFP-AtALA1 colocalized with the EE indicator RFP-RabA1d. Scale bar, $10 \mu \mathrm{m}$. $\mathbf{f}$ EGFP-AtALA1 colocalized with the LE indicator RFPRabF2a in root hair cells of transgenic Arabidopsis containing proAtALA1::EGFP-AtALA1 and 35S::RFP-RabF2a. Scale bar, $10 \mu \mathrm{m}$. $\mathbf{g}$ Inhibition of TyrA23 (tyrphostin A23), BFA (brefeldin A), and Wm (wortmannin) on the transport of DON5-FAM to vacuoles. Mock, wild-type seedlings were treated with $\operatorname{DON}^{5-F A M}(9 \mu \mathrm{g} / \mathrm{mL})$ and FM4-64 $(8 \mu \mathrm{M})$ for $12 \mathrm{~h}$. TyrA23, BFA and Wm, Mock with TyrA23 $(50 \mu \mathrm{M}), \mathrm{BFA}(50 \mu \mathrm{M})$ or Wm (33 $\left.\mu \mathrm{M}\right)$ for $12 \mathrm{~h}$. Scale bar, $5 \mu \mathrm{m}$. Experiments were independently repeated at least three times. The fluorescence density profile was measured along the dotted lines using ImageJ. The values of the Pearson correlation coefficient (Rp) and the Spearman correlation coefficient (Rs) show the extent of colocalization between the two target molecules. The values range between +1 (positive correlation) and -1 (negative correlation).

also increased the resistance of tobacco to CIA (Supplementary Fig. 6b, c).

We further tested the resistance of AtALA7 transgenic Arabidopsis to a strong pathogenic strain of $V$. dahliae L2-1. After inoculation, typical symptoms of Verticillium wilt appeared in the wild-type control, whereas severe symptoms were observed in the ala7 and ala7-24 mutants; in contrast, AtALA7-overexpressing lines showed less-severe disease (Fig. 4f, g). The relative fungal biomass of $V$. dahliae in the 35S::AtALA7 line (OE9 and OE-12) was lower than that of the wild-type and mutant plants (Fig. 4h). Likewise, 35S::AtALA7 tobacco also displayed a significantly lower disease index than the wild-type (Fig. 4i, j). These results suggest that the overexpression of AtALA7 can increase plant resistance against different $V$. dahliae strains (e.g., L2-1 and V991).

Overexpression of AtALA1 promotes DON transport to vacuoles and increases the resistance of Arabidopsis to DON and F. graminearum. To confirm the role of AtALA1 in DON detoxification, the gene was overexpressed in Arabidopsis. Similar to AtALA7, neither upregulation nor knockout of AtALA1 significantly affected the growth of the plants (Fig. 5a, b, and Supplementary Fig. 5e, g, h). Transgenic lines growing on the DONcontaining medium exhibited better plant growth with more biomass, while those of ala1 and ala1-8 mutants displayed poorer growth and less biomass relative to the wild-type control (Fig. 5a-d). In addition, the 35S::AtALA1 lines exhibited a stronger ability to transport $\mathrm{DON}^{5-\mathrm{FAM}}$ into vacuoles than wildtype (Fig. 5e, f).

The DON-producing pathogen $F$. graminearum was then used to test disease resistance via the Fusarium-Arabidopsis floral pathosystem ${ }^{90}$. Six days after inoculation with $F$. graminearum spores, mycelia emerged on the surface of buds. More mycelia were observed on the buds of mutant plants (ala1 and ala1-8) compared with those of the wild-type, while the 35S::AtALA1 Arabidopsis (OE-15 and OE-25) buds had remarkably fewer mycelia (Fig. 5g). Nine days after inoculation, severe disease symptoms of $F$. graminearum, such as brown tissue discoloration and aerial mycelium on the surface of siliques, were observed on mutant plants (ala1 and ala1-8) and wild-type, whereas the disease symptoms of $35 S:: A t A L A 1$ lines were mild (Fig. $5 \mathrm{~g}-\mathrm{i})$. The F. graminearum abundance in ala1 mutant plants was significantly higher than that in wild-type; in contrast, this abundance was much lower in 35S::AtALA1 lines (Fig. 5j). Determination of the DON concentration in siliques harvested from these infected plants showed that the content of AtALA1 lines OE-15 and OE25 was $1.50 \pm 0.25 \mathrm{ng} / \mathrm{g}$ and $1.25 \pm 0.29 \mathrm{ng} / \mathrm{g}$, respectively, which was significantly lower than that in the wild-type siliques $(3.53 \pm 0.75 \mathrm{ng} / \mathrm{g}$; Fig. $5 \mathrm{k})$. However, the concentrations in the ala1 and ala1-8 siliques $(6.19 \pm 0.56 \mathrm{ng} / \mathrm{g}$ and $7.22 \pm 0.54 \mathrm{ng} / \mathrm{g})$ were largely higher than that in the wild-type (Fig. 5k). These data demonstrate the significant efficacy of AtALA1 in DON detoxification.

Heterologous overexpression of AtALA1 enhances the resistance of maize to ear rot and significantly reduces the DON concentration in seeds. To test the DON detoxification ability of AtALA1 in monocot crops, we expressed AtALA1 under the control of the ubiquitin promoter in maize. Three transgenic Ubi1::AtALA1 lines (\# 4, \# 13, and \# 15) with relatively high levels of AtALA1 transcription were used in this study (Supplementary Fig. $7 \mathrm{a}, \mathrm{b}$ ). The transgenic maize plants were indistinguishable from the wild-type in plant growth and seed size (Supplementary Fig. $7 \mathrm{c}-\mathrm{h}$ and Supplementary Table 2). DON ranging from 30 to $90 \mu \mathrm{M}$ inhibited radicle elongation in a dose-dependent manner (Supplementary Fig. 6d, e); thus, we treated seeds of transgenic maize with $60 \mu \mathrm{M}$ DON to investigate this resistance. Although the radicle elongation of Ubi1::AtALA1maize seedlings $\left(\mathrm{T}_{3}\right)$ was inhibited by DON, the extent of inhibition was much weaker than that of the wild-type (Fig. 6a, b). The DON ${ }^{5-F A M}$ accumulation assay showed that a large amount of fluorescent signal was compartmentalized in vacuoles of the Ubi1::AtALA1 lines (\# 4), which was much stronger than that in the vacuoles of the wildtype (Fig. 6c, d).

Next, F. graminearum was inoculated into the coleoptiles of 6day-old seedlings. Six days later, a large area of wild-type coleoptiles became dark brown, and aerial mycelia were visible on the surface. In contrast, the disease severity on Ubi1::AtALA1 coleoptiles was strikingly lower than that on the control (Fig. 6e, f). Then, spores of $F$. graminearum $\left(1.3 \times 10^{8}\right.$ spores $\left./ \mathrm{mL}\right)$ were inoculated on young silks by spraying. Forty days later, the top part (nearly one-third) of the wild-type cobs became dark brown, and some cobs even began to rot. White mycelia appeared not only on the top part of the cob but also on the middle part. In contrast, in Ubi1::AtALA1 transgenic maize (\# 4, \# 13, and \# 15), the disease symptom was observed just at the very top region of the cobs, and no mycelia were found on the ears (Fig. 6g). Statistically, the disease incidence rate and disease area were significantly decreased in Ubi1::AtALA1 transgenic plants compared with the wild-type (Fig. 6h, i). Notably, the DON concentration was also decreased in transgenic maize grains. For example, in lines \# 4 and \# 15, the DON contents were $62.6 \pm 19.8 \mathrm{ng} / \mathrm{g}$ and $51.8 \pm 2.8 \mathrm{ng} / \mathrm{g}$, respectively, which were significantly lower than those in wild-type grains $(122.3 \pm 15.3 \mathrm{ng} /$ g; Fig. 6j). These data showed that heterologous expression of AtALA1 significantly increased the resistance of maize to $F$. graminearum and dramatically decreased the DON content in seeds. 

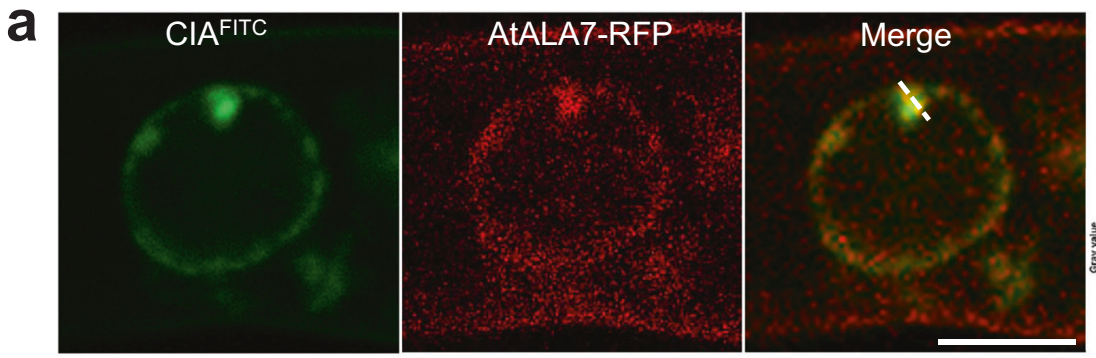

$\mathrm{Rp}=0.83 \pm 0.08$

Rs $=0.82 \pm 0.06$

b
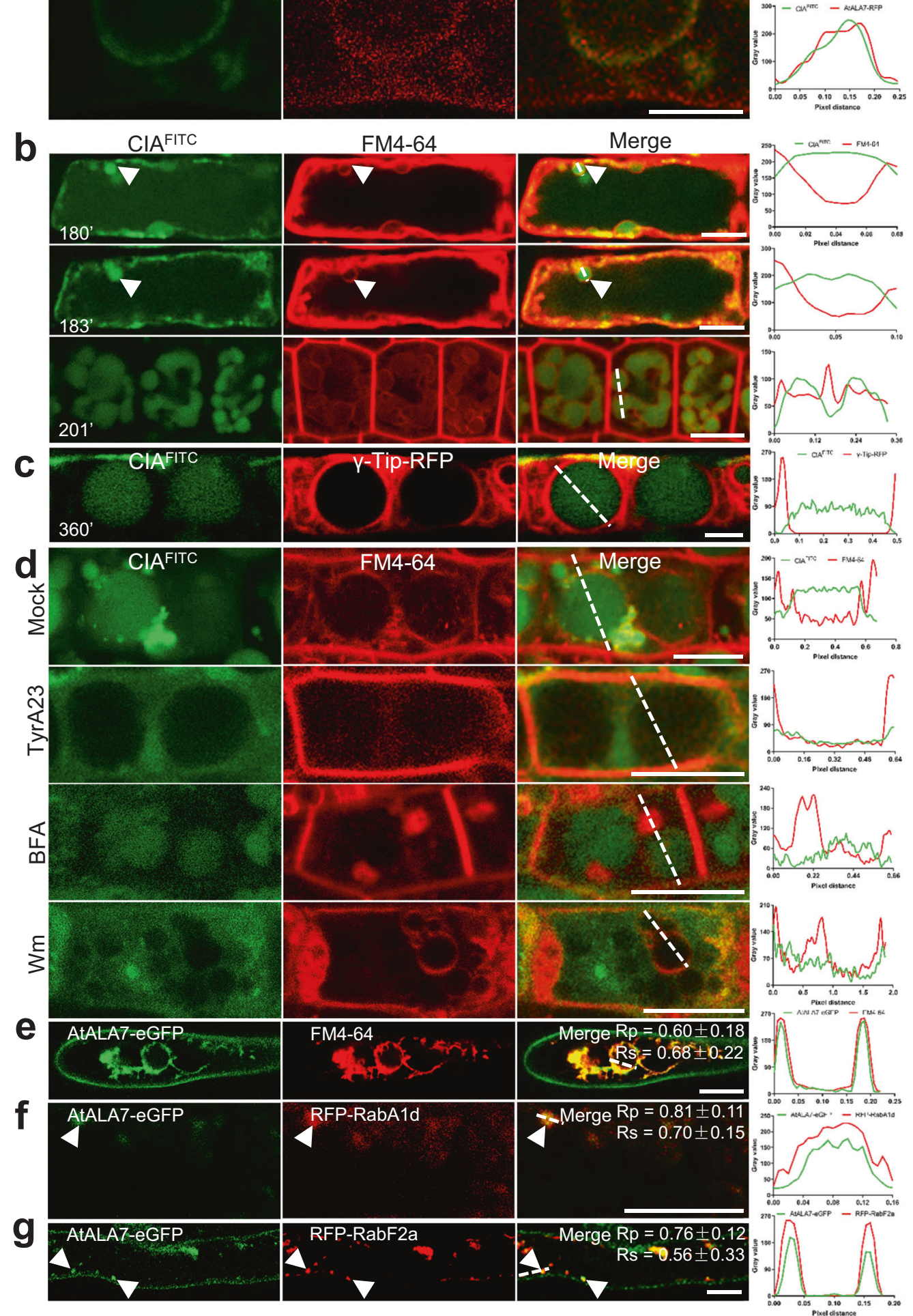

\section{Discussion}

Based on their infection and feeding strategy, plant pathogens can be divided into three major groups: biotrophs, necrotrophs, and hemibiotrophs. Biotrophic pathogens colonize living tissues without killing their hosts, whereas necrotrophic pathogens kill host cells to obtain nutrients from dead tissues; hemibiotrophic pathogens start infection as a biotroph and then live as saprophytes on dead tissues ${ }^{2,91,92}$. When plants are infected by biotrophic pathogens, hypersensitive responses (HR), including oxidative bursts, are induced to trigger programmed cell death (PCD), which in turn confines the infection to dead cells. In contrast, necrotrophic fungi usually produce toxins to subvert 
Fig. 3 CIAFITC is transported via the EE and LE into lytic vacuoles. a CIAFITC colocalized with AtALA7-RFP in vesicles and vacuoles of the root hair 35S::AtALA7-RFP seedlings were treated with CIA FITC $(15 \mu \mathrm{g} / \mathrm{mL})$ for $6 \mathrm{~h}$. Scale bar, $5 \mu \mathrm{m}$. b CIA FITC and FM4-64 fluorescence in root cells of wild-type Arabidopsis at $180 \mathrm{~min}, 183 \mathrm{~min}$, and $201 \mathrm{~min}$ after treatment with CIA $\mathrm{FITC}(15 \mu \mathrm{g} / \mathrm{mL})$ and FM4-64 $(8 \mu \mathrm{M})$. Scale bar, $10 \mu \mathrm{m}$. The triangle indicates the location of CIA ${ }^{\text {FITC }}$ aggregates. c Distribution of CIA FITC in root cells of transgenic Arabidopsis 35S:: $\gamma$-Tip-RFP. Seedlings were treated with CIA $\mathrm{mL}$ ) for $6 \mathrm{~h}$; CIA FITC fluorescence mainly accumulated in lytic vacuoles. Scale bar, $10 \mu \mathrm{m}$. $\mathbf{d}$ Inhibition of TyrA23, BFA, and Wm on the accumulation of CIA FITC in vacuoles of root cells. Arabidopsis wild-type seedlings were treated with $5 \mu \mathrm{g} / \mathrm{mL} \mathrm{CIAFITC}$ and $8 \mu \mathrm{M}$ FM4-64 (Mock), and extra TyrA23 ( $50 \mu \mathrm{M}$ ), BFA $(50 \mu \mathrm{M})$ or $\mathrm{Wm}(33 \mu \mathrm{M})$ for $6 \mathrm{~h}$. Scale bar, $10 \mu \mathrm{m}$. e AtALA7-eGFP was localized to the PM and tonoplasts in root hair cells of proAtALA7::AtALA7eGFP transgenic Arabidopsis, which were stained with FM4-64 $(8 \mu \mathrm{M})$. proAtALA7, the native promoter of AtALA7. Scale bar, 10 $\mu$ m. f AtALA7-eGFP colocalized with the EE indicator RFP-RabA1d in root hair cells of transgenic Arabidopsis containing proAtALA7::AtALA7-eGFP and 35S::

RFP-RabA1d. Scale bar, $10 \mu \mathrm{m}$. g AtALA7-eGFP colocalized with the LE marker RFP-RabF2a in root hair cells of transgenic Arabidopsis containing proAtALA7::AtALA7-eGFP and 35S:::RFP-RabF2a. Scale bar, $10 \mu \mathrm{m}$. Experiments were independently repeated at least three times. The fluorescence density profiles were measured along the dotted lines using ImageJ. The values of the Pearson correlation coefficient (Rp) and the Spearman correlation coefficient (Rs) show the extent of colocalization between the two target molecules. The values range between +1 (positive correlation) and -1 (negative correlation).

host defenses, such as HR, to accelerate the PCD of host cells, thus benefiting their colonization in plants ${ }^{2}$. Pathogenesis is more complicated for hemibiotrophic pathogens: they can generate low levels of toxins to suppress PCD, thus facilitating pathogen colonization and infection during the initial biotrophic phase ${ }^{93}$. They can also produce high levels of toxins to stimulate cell death, thus releasing nutrients to facilitate necrotrophic fungal growth during the necrotrophic phase $\mathrm{s}^{35,94,95}$. The complicated pathogenic process makes it difficult to control hemibiotrophic pathogens. As toxins play a key role in both biotrophic and necrotrophic pathogenicity, detoxification is, therefore, a logical strategy for controlling these diseases.

Although the development of resistance to FHB by chemical modification/inactivation of trichothecenes have been achieved in wheat and barley ${ }^{42}$, this strategy has apparent limitations. One drawback of this strategy is the potential undesirable side effects on plant growth or development, resulting from the manipulation of metabolism. For example, glycosylation mediated by glycosyltransferase has been utilized in plants for DON detoxification ${ }^{36,96}$. Since some glycosyltransferases have a wide range of substrates, including plant hormones, their overexpression in plants may interfere with hormone homeostasis, thus resulting in abnormal growth ${ }^{36,41,97}$. For instance, although the overexpression of Arabidopsis glycosyltransferase DOGT1, which converts toxic DON to nontoxic D3G, increased the resistance of Arabidopsis to DON, transgenic Arabidopsis displayed a brassinosteroid deficiency-like dwarf phenotype due to the formation of brassinosteroid glucosides ${ }^{36,41}$. More importantly, glycosylated Fusarium toxins can be reversed in animals during digestion ${ }^{42}$. In fact, all modified forms of Fusarium toxins likely have the same toxicity to human and animal health as their parent compounds ${ }^{43}$. In vesicle-mediated detoxification, in contrast, toxins are wrapped into vesicles surrounded by membranes and then transported into vacuoles where the toxins are compartmentalized and degraded; thus, there is no chance for the toxins to be in contact with their targets. In our experiments, no significant alterations in plant growth or development were observed in the transgenic plants overexpressing AtALA1/ AtALA7. Most importantly, the resistance to F. graminearum and $V$. dahliae is significantly increased, and the DON contamination in maize grains is largely reduced.

Our data show that the entry speed of CIA into the cytosol is faster than that of DON (Supplementary Fig. 3e, f). Because of its lipophilic character and small molecular weight (176.21 Da), CIA can cross the plasma membrane and enter the cell (Figs. 1i, 3b and Supplementary Fig. $2 \mathrm{~g}, 3 \mathrm{e}$ ), resulting in wilt symptoms in plants $^{98}$ (Fig. 1f, 4a). In contrast, DON has a polar molecule structure. The ability of DON to enter cells by diffusing across the cell membrane, therefore, is very low ${ }^{99}$. The possible way of DON enters cells may be through an unknown membrane-associated passive transporter or endocytosis/pinocytosis process ${ }^{99}$. Once entering cells, the amphiphilic nature of DON can increase its cytotoxicity on the cell by interactions with a number of targets, including ribosomes ${ }^{100}$ and mitochondria ${ }^{101,102}$. Our study shows that either lipophilic CIA or amphiphilic DON can be wrapped in vesicles and transported from the PM to vacuoles; thus, the amount of toxins entering cells through other pathways is significantly reduced. The detoxification mechanism mediated by AtALA1/AtALA7 is shown in Fig. 7.

In the present study, using DON and CIA as probes, we successfully identified the Arabidopsis AtALA1 and AtALA7 responsible for the detoxification of mycotoxins in plants. We, therefore, hypothesize that cargoes transported by P4 ATPaseassociated vesicle trafficking for detoxification should be a group of molecules with similar physicochemical or biological properties. AtALA1 may be responsible for the transport of some amphiphilic molecules, such as DON, whereas AtALA7 may be responsible for the transport of lipophilic compounds, such as CIA. If this is the case, P4 ATPase(s) can potentially be utilized for detoxifying different toxins in various species. Details about what properties the compounds should have and how the P4 ATPases recognize their cargos await further investigation. In addition to AtALA1 and AtALA7, other ALAs may also participate in the detoxification of other toxins. The method described here allows us to identify more P4 ATPases that are responsible for transporting specific molecules.

In brief, our findings may help drive research on increasing plant resistance against intractable plant diseases caused by toxinproducing pathogens, such as rice sheath blight, rice blast, $\mathrm{FHB}$, and Verticillium wilt. This P4 ATPase-conferred resistance can potentially be achieved in different species against different diseases, and the detoxification strategy is also applicable to cope with mycotoxin contamination in food and feed.

\section{Methods}

Plant materials and growth conditions. The Arabidopsis thaliana T-DNA insertion mutants ala1 (salk_056947), ala1-8 (salk_002106), ala2 (salk_070975), ala3 (salk 082157), ala4 (salk 078875), ala5 (salk_056611), ala6 (salk_150173), ala7 (salk_125598), ala7-24 (salk_063917), ala8 (salk_071661), ala9 (salk_070924), ala10 (salk_107029) and ala11 (salk_056947) were obtained from the SALK collection. These mutants were genotyped using a PCR-based approach (http:// signal.salk.edu/tdnaprimers.2.html). The PCR primer sequences are given in Supplementary Table 1 .

Seeds of Arabidopsis thaliana (ecotype Columbia, Col-0) were surface-sterilized in $75 \%$ alcohol for $20 \mathrm{~min}$, rinsed 3 5 times with sterile water, and sown on MS medium (Murashige and Skoog medium, M519, Phytotech) containing 1.5\% (w/v) sucrose and $1 \%(\mathrm{w} / \mathrm{v})$ gelrite. Plants were incubated at $4{ }^{\circ} \mathrm{C}$ for $2 \mathrm{~d}$ in the dark and then transferred to a growth chamber at $21 \pm 2{ }^{\circ} \mathrm{C}$ with a $16 \mathrm{~h}$ light $/ 8 \mathrm{~h}$ dark photoperiod.

Tobacco was grown in a greenhouse at $25^{\circ} \mathrm{C}$ under an $18 \mathrm{~h}$ light $/ 6 \mathrm{~h}$ dark photoperiod. Agrobacterium tumefaciens strain EHA105 was used to transform Nicotiana tabacum $c v$. Xanthi ${ }^{103}$. The maize cultivar Hi $\mathrm{II}^{104}$ was used for genetic transformation and DON inhibition assays. Seeds were incubated in sterile water for $24 \mathrm{~h}$ in the dark and placed between two sheets of sterile Whatman filter paper 
a

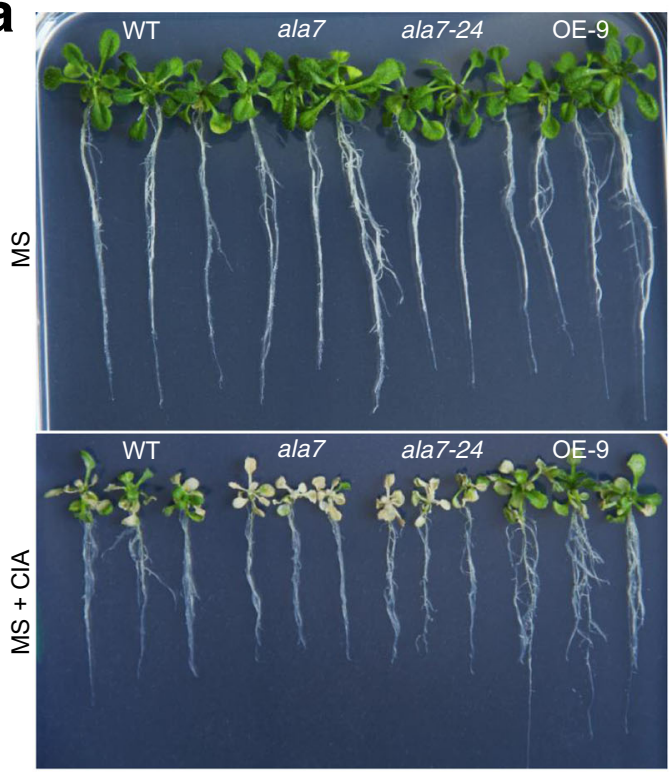

d

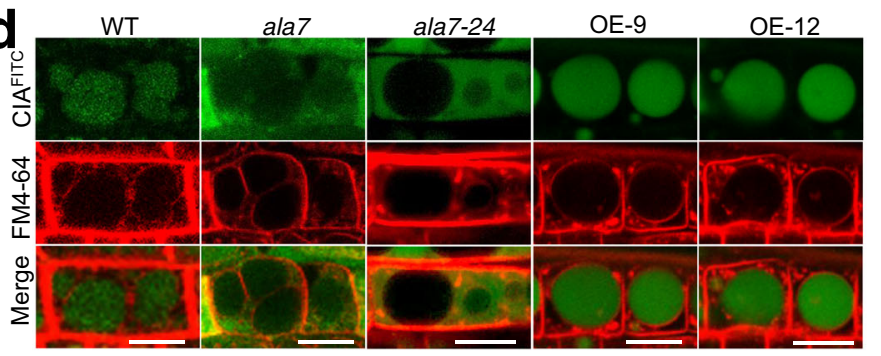

f

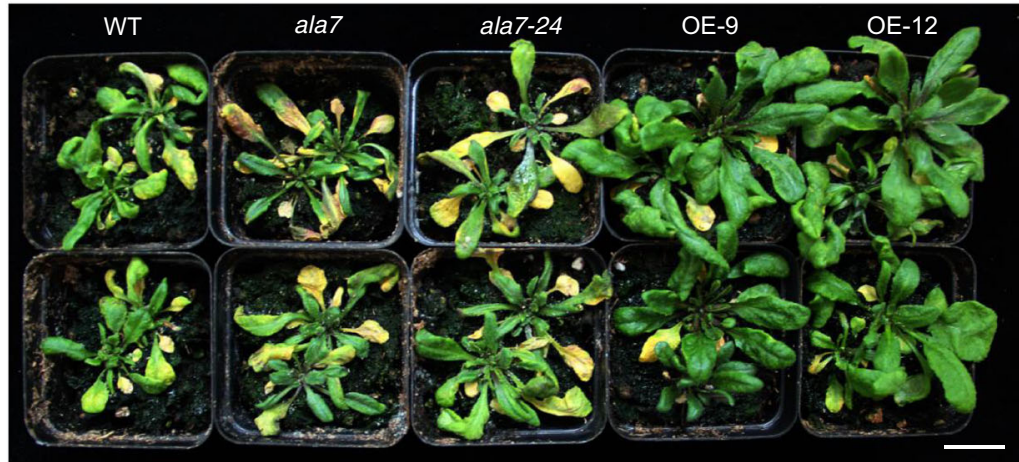

g

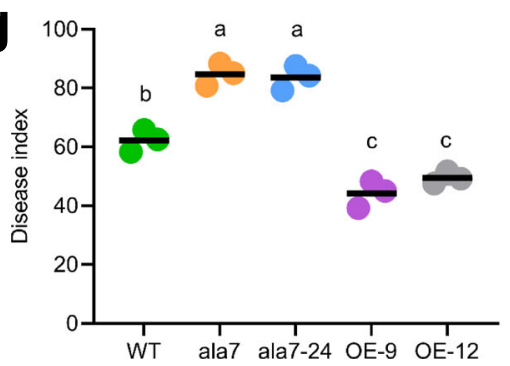

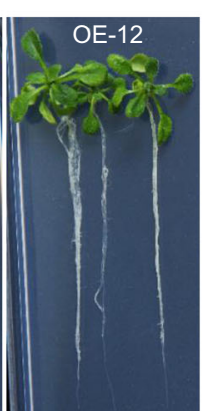

C.
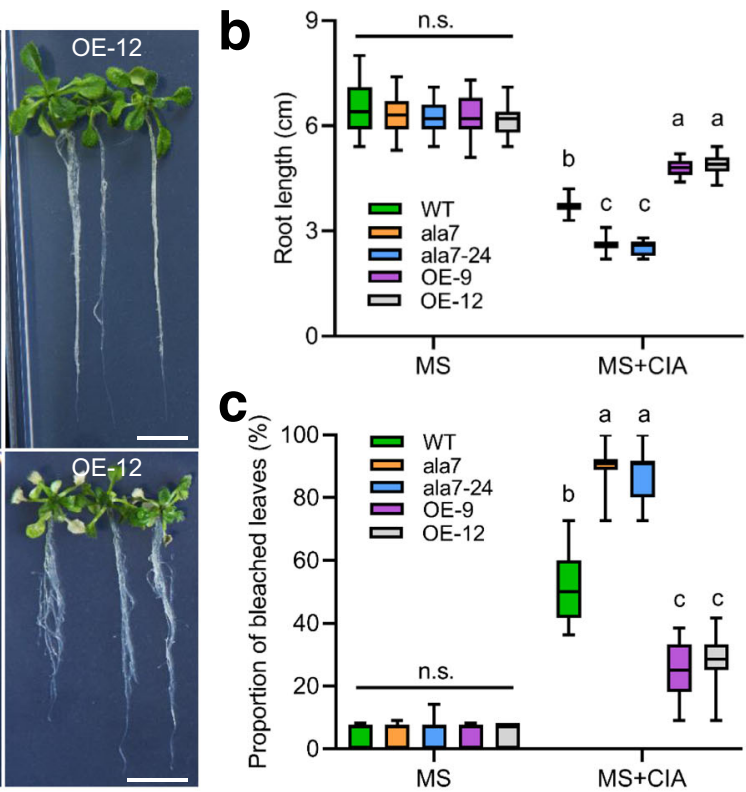

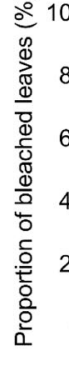

e

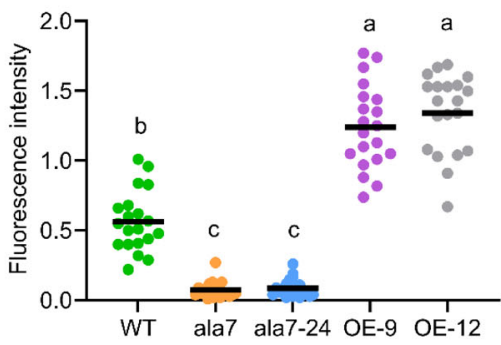

with $5 \mathrm{~mL}$ of sterile water inside a Petri dish. Seeds were incubated for $5 \mathrm{~d}$ in total darkness at $25^{\circ} \mathrm{C}$ to ensure germination.

Gene cloning and transgenic plant production. cDNA fragments were cloned into a pLGN vector modified from pCambia $2300^{105}$. In the T-DNA region of pLGN, there is a neomycin phosphotransferase II (NPTII) selection marker fused with a GUS reporter gene under the control of a $35 S$ promoter. For the 35S::AtALA1 constructs, the coding sequence of AtALA1 was cloned from
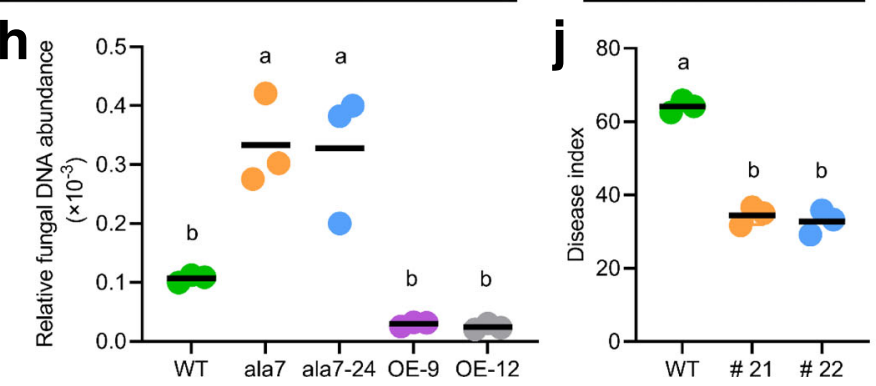
Fig. 4 Overexpression of AtALA7 promotes the transport of CIA to vacuoles and enhances the resistance of Arabidopsis and tobacco to CIA and V. dahliae. a In the presence of CIA, the growth of wild-type seedlings was significantly inhibited, and the inhibition was more severe in the ala7 mutant; in contrast, 35S::AtALA7 seedlings showed resistance to the toxin. Seedlings were grown on MS media containing CIA (100 $\mu \mathrm{g} / \mathrm{mL})$ for $9 \mathrm{~d}$. WT, wild-type; ala7 and ala7-24, AtALA7 loss-of-function mutant; OE-9 and OE-12, 35S::AtALA7 lines. Scale bar, $1 \mathrm{~cm}$. b Root length of the wild-type, ala7 mutant and AtALA7-overexpressing seedlings with or without CIA treatment. Data are presented as box-and-whisker plots of three replicates (nine plants each). c Proportion of bleached leaves suffering from CIA treatment. Data are presented as box-and-whisker plots of three replicates (nine plants each). d Distribution of CIA FITC in root cells of wild-type, mutant (ala7, ala7-24), and AtALA7-overexpressing lines (OE-9, OE-12). Scale bar, 10 $\mu$ m. e Fluorescence intensity per vacuole in root cells of wild-type, mutant (ala7, ala7-24) and 35S::AtALA7-overexpressing plants (OE-9). Data are shown as dot plots ( $n=20$ vacuoles). Fluorescence intensity was estimated by ImageJ. f Verticillium wilt symptoms of wild-type, mutant (ala7, ala7-24), and AtALA7-overexpressing Arabidopsis lines (OE-9, OE-12). Plants were inoculated with $V$. dahliae strain L2-1 (3 mL, $2 \times 10^{8}$ spores $\left./ \mathrm{mL}\right)$ for $21 \mathrm{~d}$. Scale bar, $2 \mathrm{~cm}$. $\mathbf{g}$ Disease index of wild-type, mutant (ala7, ala7-24), and AtALA7-overexpressing lines (OE-9 and OE-12). Data are shown as dot plots of three replicates (30 plants each). $\mathbf{h}$ Relative fungal biomass of $V$. dahliae shown as dot plots of three replicates (eight plants per group). $\mathbf{i}$ Verticillium wilt symptoms of wild-type and AtALA7 transgenic tobacco lines (\# 21 and \# 22). Seedlings were inoculated with $V$. dahliae strain $V 991\left(10 \mathrm{~mL}, 2 \times 10^{8} \mathrm{spores} / \mathrm{mL}\right)$ and photographed at 3 weeks. The white arrow indicates diseased leaves. Scale bar, $2 \mathrm{~cm}$. $\mathbf{j}$ Disease index of wild-type and AtALA7 transgenic tobacco plants. Data are presented as dot plots of three replicates (30 plants each). Box-and-whisker plots show the medians (horizontal lines), upper and lower quartiles (box edges), and 1.5 $\times$ the interquartile range (whiskers). Different letters in $\mathbf{b}, \mathbf{c}, \mathbf{e}, \mathbf{g}, \mathbf{h}$, and $\mathbf{j}$ represent significant differences at $P<0.05$ by one-way ANOVA with Tukey multiple comparisons test. n.s., not significant.

tail/-R (+linker) tail and AtALA1-F (+linker) tail/-R (SalI) tail, respectively, and the resulting products were fused by overlap extension PCR to link EGFP with AtALA1 (EGFP-AtALA1). Then, the PCR product was digested with SmaI/SalI and inserted into the pLGN vector to produce the pLGN-35S::EGFP-AtALA1 cassette. For the proAtALA1::EGFP-AtALA1 construct, the promoter sequence of AtALA1 (2039 bp) was cloned and fused with EGFP-AtALA1 by overlap PCR. The resulting fragment was inserted into the pLGN vector (BamHI/HindIII) via homologous recombinase (Boer, MC001M-50 Rxns). To link the C-terminus of AtALA7 with EGFP, EGFP, and AtALA7 were cloned with primer pairs AtALA7-F (SpeI)/-R (+linker) tails and EGFP-F (+linker) tail/-R (KpnI) tail, respectively, and the resulting products were fused by overlap extension PCR to link AtALA7 and EGFP (AtALA7-eGFP). Then, the PCR product was digested with SpeI/KpnI and inserted into the pLGN vector to form the pLGN-35S::AtALA7-eGFP cassette. For the proAtALA7::AtALA7-eGFP construct, the promoter sequence of AtALA7 (2053 bp) was cloned and fused with AtALA7-eGFP by overlap PCR. The resulting fragment was inserted into the $\mathrm{pLGN}$ vector (BamHI/HindIII) via homologous recombinase (Boer, MC001M-50 Rxns). For organelle marker protein construction, the CDS of Pip2a (At3g53420), RabA1d (At4g18800), RabF2a (At5g45130), and $\gamma$-Tip (At2g36830) were fused with a linker and RFP by overlap PCR. The resulting fragments were inserted into a digested pLGN vector $(B a m H I / E c o R I$ for Pip2a/ $\gamma$ Tip and BamHI/KpnI for RabAld/RabF2a) via homologous recombinase. For the Ubi1::AtALA1 construction, the coding sequence of AtALA1 was cloned with primer pairs of AtALA1-F (+Ubil, StuI) tail/-R (+Ubil, KpnI) tail, and the PCR product was digested with $S t u \mathrm{I} / K p n$. The resulting fragment was inserted into a modified pLGN vector ( $35 \mathrm{~S}$ was replaced by the ubiquitin promoter Ubil). PCR primer sequences are shown in Supplementary Table 1.

Maize transformation was conducted using particle bombardment ${ }^{106}$. Arabidopsis transformation was performed by the floral-dip method ${ }^{107}$ using Agrobacterium tumefaciens strain GV3101. Homozygous transgenic plants were screened by kanamycin $(50 \mu \mathrm{g} / \mathrm{mL})$ and PCR.

RNA extraction and quantitative (real-time) reverse transcription PCR (qRTPCR). RNA was extracted using the EASY Spin Plant RNA Kit (Aidlab, China) according to the manufacturer's instructions. Approximately $1.0 \mu \mathrm{g}$ of RNA was used for cDNA synthesis with a RevertAid First Strand cDNA Synthesis Kit (Fermentas, Canada). A 10- $\mu \mathrm{L}$ reaction mixture containing cDNA and SYBR Green Super-mix (Bio-Rad) was used for qRT-PCR following the manufacturer's instructions. The thermal cycling program consisted of an initial denaturation at $95^{\circ} \mathrm{C}$ for $3 \mathrm{~min}$, followed by 40 cycles of $95^{\circ} \mathrm{C}$ for $15 \mathrm{~s}, 58^{\circ} \mathrm{C}$ for $20 \mathrm{~s}$ and $72^{\circ} \mathrm{C}$ for $30 \mathrm{~s}$. Data were analyzed by CFX Manager 3.1 software (Bio-Rad, USA). Each test was repeated at least three times. AtActin2, ZmEF1 $\alpha$, and NtActin were used as reference genes in Arabidopsis, maize, and tobacco, respectively. The primers used for qRT-PCR are listed in Supplementary Table 1.

Subcellular localization. The colocalization of AtALA1 and AtALA7 with different organelle markers fused with RFP was carried out by transient expression in tobacco epidermal cells or by the floral-dip method in Arabidopsis with Agrobacterium tumefaciens strain GV3101 containing EGFP-AtALA1 (or AtALA7$e G F P$ ) and organelle markers (PiP2a-RFP, RFP-RabF2a, RFP-HDEL, and RFPRabAld). For transient expression in tobacco epidermal cells, A. tumefaciens cells were pelleted by centrifugation and resuspended in infiltration solution $(10 \mathrm{mM}$ $\mathrm{MgCl}_{2}, 10 \mathrm{mM}$ MES and $0.2 \mathrm{mM}$ acetosyringone) until the $\mathrm{OD}_{600}$ reached 0.5 ; this was followed by incubation in the dark for $3 \mathrm{~h}$ before co-infiltration into leaves of $N$. benthamiana. The fluorescent signal was detected with a microscope (Leica SP8, Germany) at 48-72 $\mathrm{h}$ after co-infiltration. For the localization of AtALAl (or
AtALA7) in PM and tonoplasts, roots of EGFP::AtALA1 transgenic Arabidopsis were stained with FM4-64 ( $8 \mu \mathrm{M}, \mathrm{F} 34653$, Thermo Scientific). The colocalization of AtALA1 (or AtALA7) to the EE was observed in root hair cells of transgenic Arabidopsis containing EGFP-AtALA1 (or AtALA7-eGFP) and an EE marker (RFP-RabA1d). The colocalization of AtALA1 (or AtALA7) to the LE was observed in root hair cells of transgenic Arabidopsis containing EGFP-AtALA1 (or AtALA7eGFP) and an LE marker (RFP-RabF2a).

Pathogen inoculation and disease scoring. The wild-type $F$. graminearum strain PH-1 (3.4584, CGMCC) was cultured on potato dextrose agar (PDA, 254920, BDDifco) plates at $26^{\circ} \mathrm{C}^{108}$. Falcate conidia were collected from PDA plates, transferred into liquid mung bean (Vigna radiata) medium ${ }^{109}$, and cultivated at $26^{\circ} \mathrm{C}$ for $3 \mathrm{~d}$ with shaking at $200 \mathrm{rpm}$. The conidial suspension was filtered through fourlayer gauze and centrifuged at $5000 \times g$ for $10 \mathrm{~min}$. The harvested conidia were resuspended in sterile water. The suspension was used for inoculation within $2 \mathrm{~h}$

Arabidopsis plants with both open flowers on the terminal inflorescence and two to three developing siliques were used for inoculation assays ${ }^{90}$. In brief, the $F$. graminearum strain $\mathrm{PH}-1^{108}$ spores suspension $\left(1.3 \times 10^{8}\right.$ spores $\left./ \mathrm{mL}\right)$ was sprayinoculated into the inflorescence until droplet run-off occurred from the inflorescence. Control plants were inoculated in the same way using deionized water. The inoculated plants were then kept in large plastic propagators at $100 \%$ relative humidity for the next 6 days. For the first 2 days, the chambers were shaded with capillary matting to exclude light. Disease scoring was assessed as described previously ${ }^{90}$ with slight modification. In brief, three separate floral subcomponents, namely, flowers (F) that were either open flowers or closed buds at inoculation, new siliques (NS) that were fully open flowers at inoculation, and older siliques (OS) already present at inoculation, were considered. An increasing numerical score was used to quantify the abundance of aerial mycelium on a tissue surface $(0,1)$, as well as the increased severity of the disease symptoms visible on plant tissue as the invasion process progressed (3-7). Intermediate scores of 2 and $4(\mathrm{~F})$ and 2, 4, and 6 (NS and OS) were reserved for when all the tissue on a single plant exhibited the disease phenotype described for the preceding score. The final

Fusarium-Arabidopsis disease (FAD) value was calculated by the addition of the three subcomponent scores, $\mathrm{FAD}=\mathrm{F}+\mathrm{NS}+\mathrm{OS}$.

For maize ear inoculation, 3-day-old silk ears of maize plants were sprayinoculated with fresh spores suspension $\left(2 \mathrm{~mL}, 5 \times 10^{5}\right.$ spores $\left./ \mathrm{mL}\right)$ of $F$. graminearum strain PH-1. After inoculation, the maize ears were covered with black bags and kept at $95 \%$ humidity for $48 \mathrm{~h}$. The disease was rated by the percentage of infected seeds at 40 dpi. For maize coleoptile inoculation, 6-day-old seedlings were used for the coleoptile infection assay according to Zhang et al. ${ }^{109}$ with modifications. In brief, 6 days after seed sowing, the top $2-3 \mathrm{~mm}$ of the coleoptiles were removed, and the seedlings were drop-inoculated with $8 \mu \mathrm{L}$ of fresh spores suspension $\left(7.5 \times 10^{7}\right.$ spores/ $\mathrm{mL}$ ) of $F$. graminearum strain $\mathrm{PH}-1$ for $6 \mathrm{~d}$. Mock-inoculated coleoptiles (with water) served as controls. After inoculation, the inoculated maize seedlings were grown in a growth chamber at $25^{\circ} \mathrm{C}$ and $95 \%$ humidity for $48 \mathrm{~h}$.

For $V$. dahliae inoculation, Arabidopsis and tobacco seedlings were inoculated with $V$. dahliae strains L2-1 and V991, respectively. The inoculation procedure was as follows: at least 50 seedlings of each construct were dipped into a spore suspension liquid containing $2 \times 10^{8}$ spores $/ \mathrm{mL}$. The disease index (DI) of Verticillium wilt was scored using at least 50 plants per treatment. The severity of disease symptoms was classified as level $0-4$. Here, 0 means no visible wilting or chlorosis symptoms ${ }^{110} .1$ means $0-25 \%$ (inclusive) of true leaves wilted, chlorosis or dropped off; 2 means $25-50 \%$ (inclusive) of true leaves wilted, chlorosis or dropped off; 3 means $50-75 \%$ (inclusive) of true leaves wilted, chlorosis or dropped off; and 4 means $75-100 \%$ (inclusive) of true leaves wilted, chlorosis or dropped off. 
a
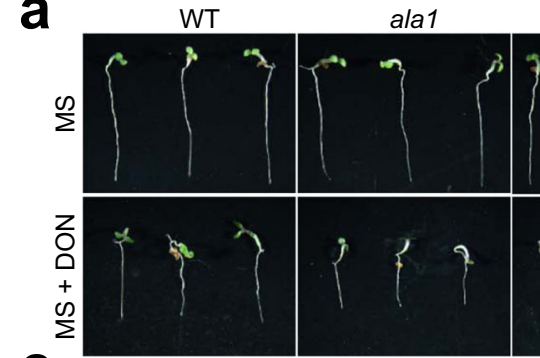

c

$$
\begin{gathered}
7 \\
\text { O } \\
+ \\
\text { c } \\
\text { s }
\end{gathered}
$$

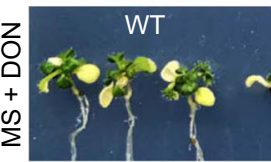

e

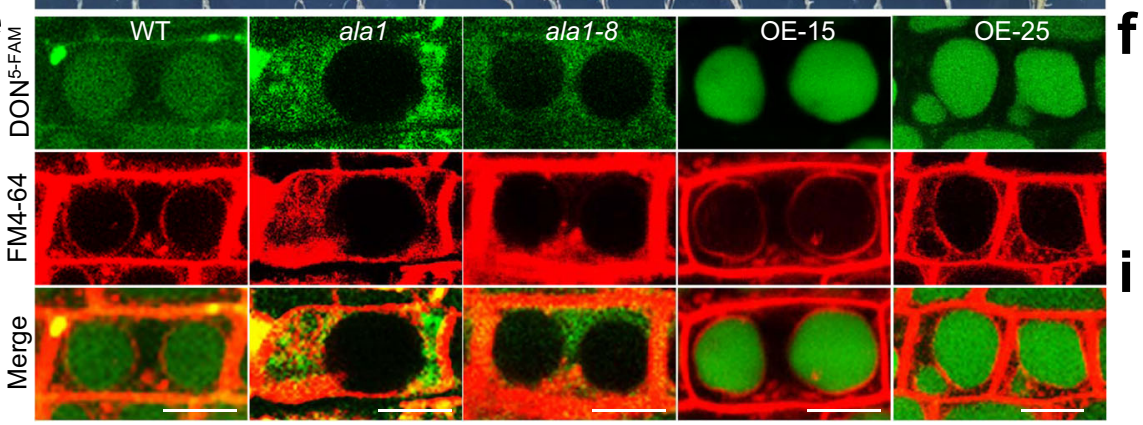

g

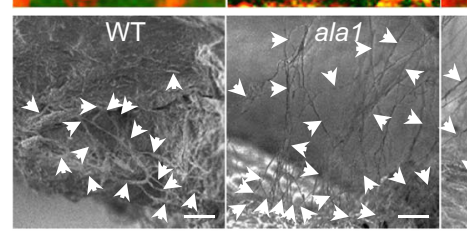

h

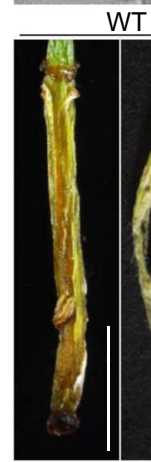

WT

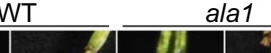

ala1-8
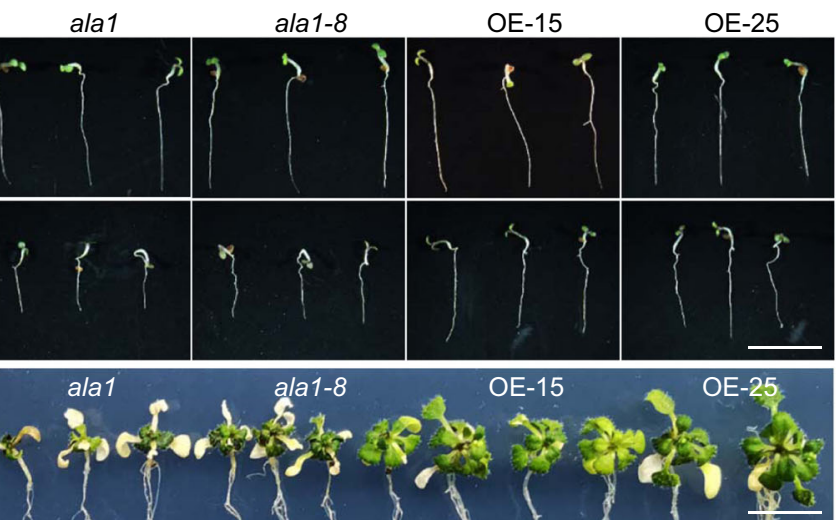

d

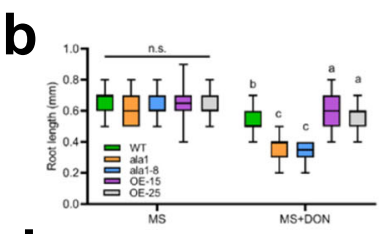

d
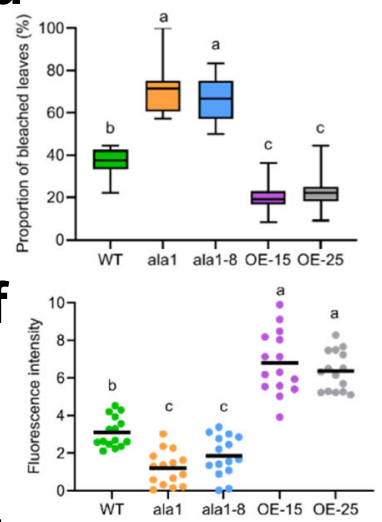

is

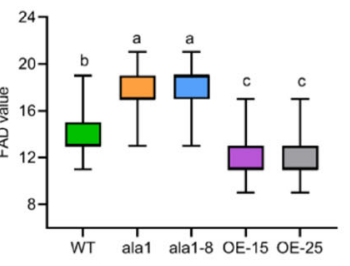

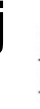
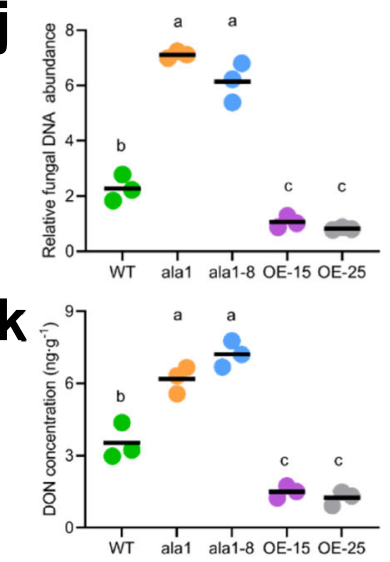

Fig. 5 Overexpression of AtALA1 promotes the transport of DON to vacuoles and increases the resistance of Arabidopsis to DON and F. graminearum. a DON resistance assay of wild-type, mutant (ala1, ala1-8), and 35S::AtALA1-overexpressing lines (OE-15 and OE-25). Two-day-old seedlings were treated with DON $(1 \mu \mathrm{g} / \mathrm{mL})$ for $5 \mathrm{~d}$. WT, wild-type Arabidopsis; ala1 and ala1-8, AtALA1 loss-of-function mutant; OE-15 and OE-25, transgenic 35S::AtALA1 Arabidopsis lines. Scale bar, $5 \mathrm{~mm}$. b Root length of plants with or without DON treatment. The results are shown with box-and-whisker plots of three replicates (12 plants each). c DON resistance assay of wild-type, mutant (ala1, ala1-8), and 35S::AtALA1-overexpressing lines (OE-15 and OE-25). Six-dayold seedlings were transferred to MS media containing DON $(50 \mu \mathrm{g} / \mathrm{mL})$ for $12 \mathrm{~d}$. Scale bar, $20 \mu \mathrm{m}$. d Proportion of bleached leaves resulting from DON treatment. Data are presented as box-and-whisker plots of three replicates (eight plants each). e Distribution of DON ${ }^{5-F A M}$ in root cells of wild-type, mutant (ala1, ala1-8), and AtALA1-overexpressing lines (OE-15, OE-25). Six-day-old seedlings were treated with DON 5 -FAM (9 $\mu \mathrm{g} / \mathrm{mL})$ and FM4-64 (8 $\mu \mathrm{M})$ for $12 \mathrm{~h}$. Scale bar, $10 \mu \mathrm{m}$. f Fluorescence intensity of DON5-FAM in root cells of wild-type, mutant (ala1, ala1-8), and AtALA1-overexpressing lines (OE-15, OE-25). The results are shown with dot plots $(n=15$ vacuoles). $\mathbf{g} F$. graminearum mycelia on the surface of buds at 6 dpi (days post-inoculation). Plants were spray-inoculated with F. graminearum $\left(1.3 \times 10^{8}\right.$ spores $\left./ \mathrm{mL}\right)$. Arrows indicate the hyphae or spores of $F$. graminearum. Scale bar, $20 \mu \mathrm{m}$. The experiment was repeated three times independently with similar results. $\mathbf{h}$ Symptoms of $F$. graminearum on siliques ( 9 dpi) of wild-type, mutant (ala1, ala18), and AtALA1-overexpressing lines (OE-15, OE-25). Plants were spray-inoculated with F. graminearum $\left(1.3 \times 10^{8} \mathrm{spores} / \mathrm{mL}\right)$. Scale bar, $1 \mathrm{~mm}$. i Disease severity estimated by the FAD (Fusarium-Arabidopsis disease) value ${ }^{90}$. The results are shown with box-and-whisker plots of three replicates (nine plants each). j Real-time PCR analysis of the DNA abundance of $F$. graminearum in flowers at 9 dpi. Data are presented as dot plots of three replicates (12 plants each). The copy number of the F. graminearum TRI6 gene was estimated relative to that of the Arabidopsis actin2 gene. $\mathbf{k}$ DON concentration from F. graminearum-infected siliques of wild-type, mutant (ala1, ala1-8), and AtALA1-overexpressing lines (OE-15, OE-25). The results are shown as dot plots of three replicates (five plants each). DON concentration was determined by LC-MS/MS. Box-and-whisker plots show the medians (horizontal lines), upper and lower quartiles (box edges), and 1.5× the interquartile range (whiskers). Different letters in $\mathbf{b}, \mathbf{d}, \mathbf{f}, \mathbf{i}-\mathbf{k}$ represent significant differences at $P<0.05$ by one-way ANOVA with Tukey multiple comparisons test. n.s., not significant. 


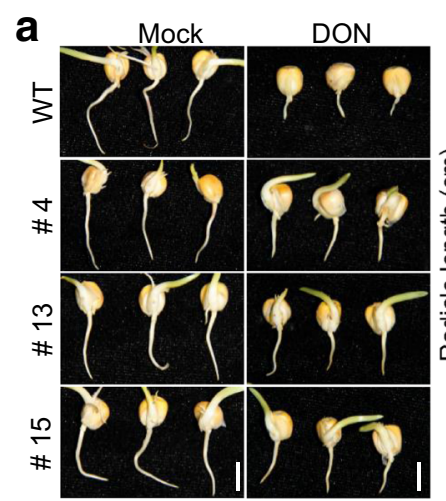

$\mathbf{e}$

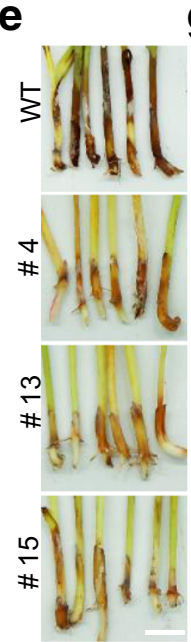

f

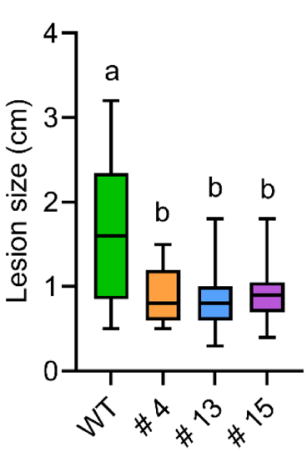

b

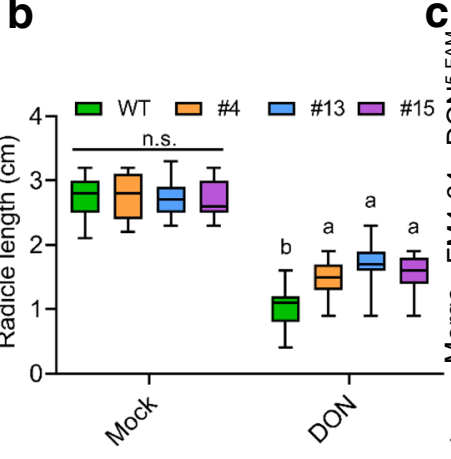

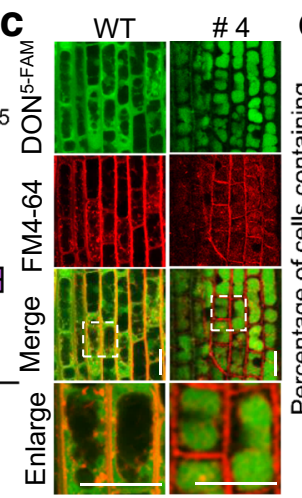
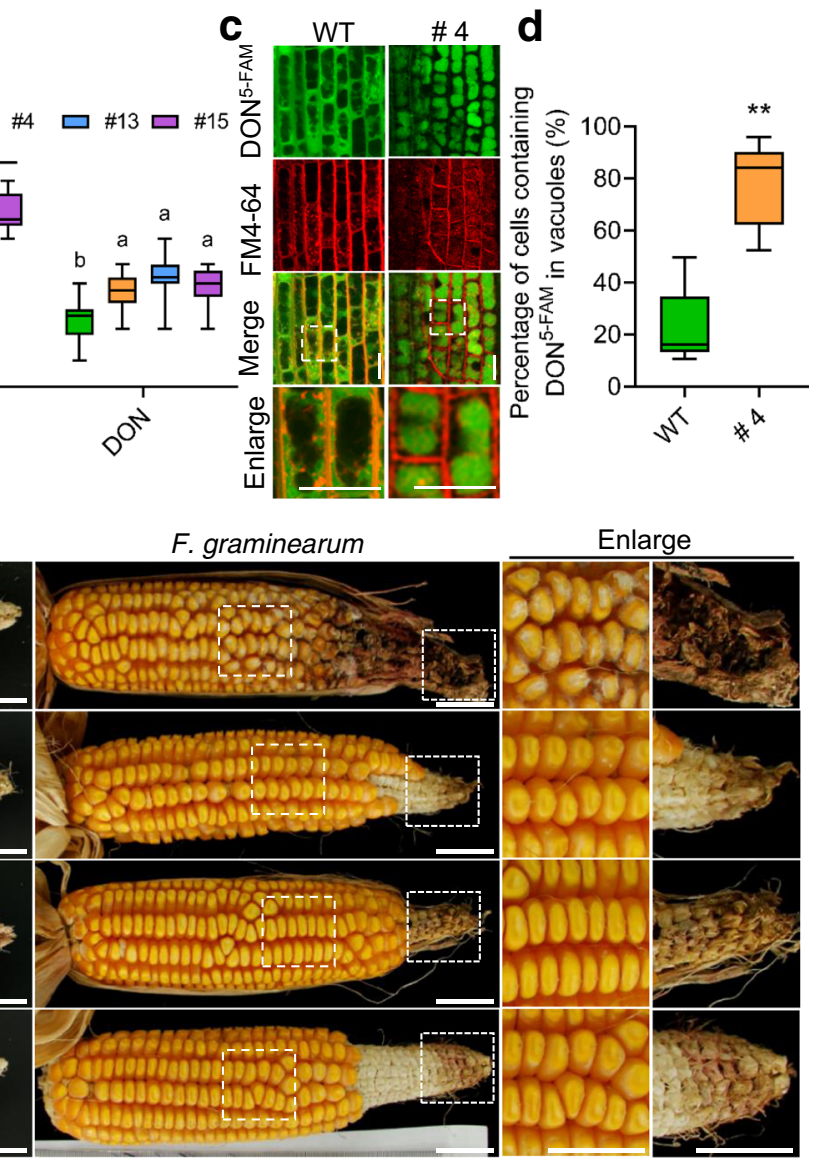
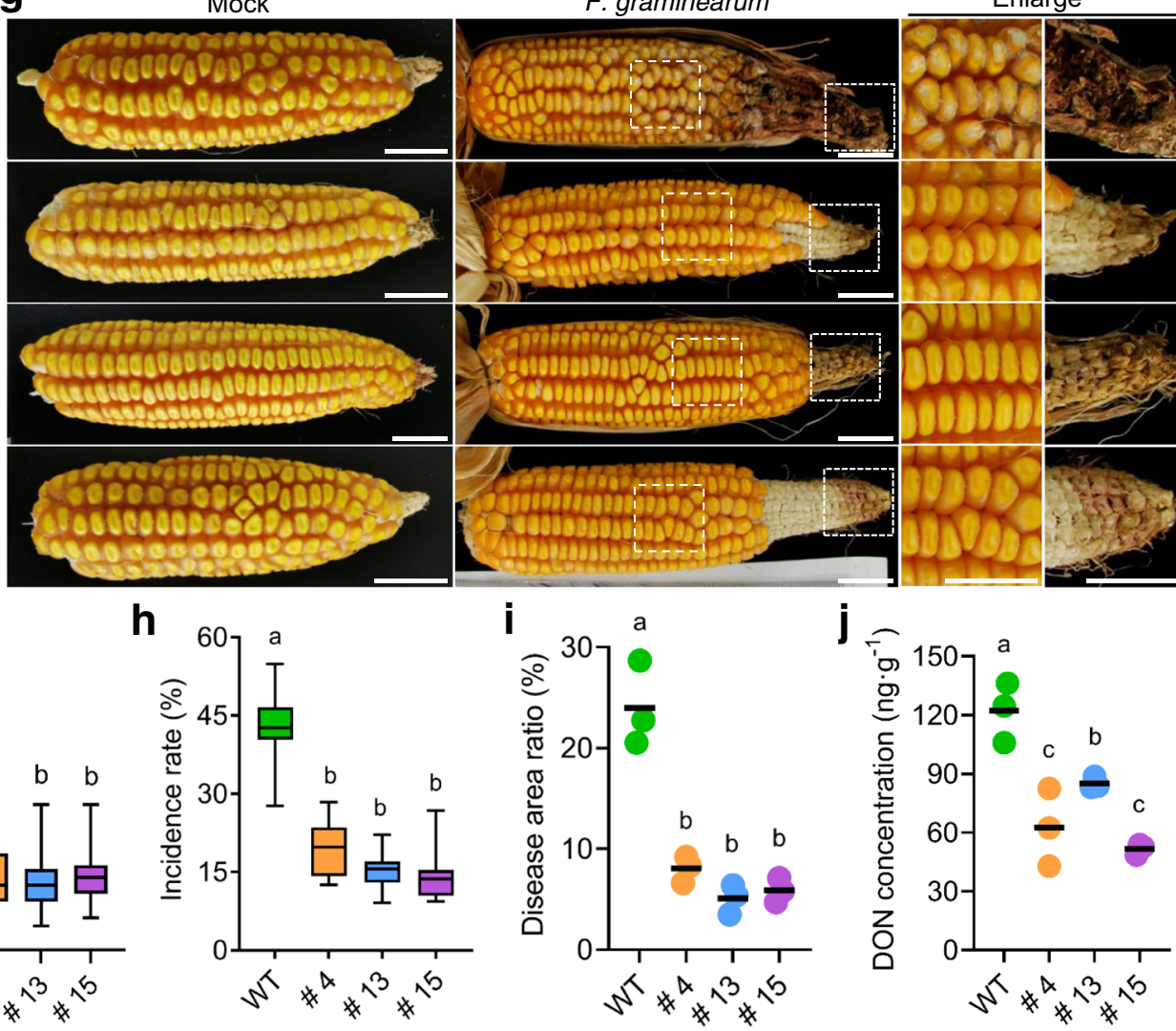

Fig. 6 Heterologous overexpression of AtALA1 in maize enhances resistance to F. graminearum and decreases DON contamination in seeds. a DON inhibition assay of Ubi1::AtALA1 transgenic lines and wild-type seeds. Seeds were soaked with DON $(60 \mu M)$ or sterilized water and then germinated in moisture chambers for $2 \mathrm{~d}$. WT, wild-type maize (cultivar Hi II); \# 4, \# 13 and \# 15, transgenic Ubi1::AtALA1 seeds ( $\left.T_{3}\right)$. Scale bar, $0.5 \mathrm{~cm}$. b Radicle length of Ubi1::AtALA1 transgenic lines and wild-type seeds in the absence or presence of DON $(60 \mu \mathrm{M})$. The results are shown with box-and-whisker plots of three replicates (5 roots each). c Distribution of DON5-FAM in root cells of Ubi1::AtALA1 transgenic lines and wild-type. Three-day-old seedlings were treated with DON ${ }^{5-F A M}(30 \mu \mathrm{g} / \mathrm{mL})$ and FM4-64 $(20 \mu \mathrm{M})$ for $12 \mathrm{~h}$. Scale bar, $20 \mu \mathrm{m}$. d Statistics of the proportion of DON ${ }^{5-F A M}$-containing vacuoles in the root cells, as shown with box-and-whisker plots of three replicates (nine roots each). ${ }^{\star \star} p<0.01$, significantly different with respect to wild-type according to Student's $t$ test (two-tailed). e Stalk rot symptoms on coleoptiles of Ubi1::AtALA1 transgenic lines and wild type. F. graminearum spores suspension ( $8 \mu \mathrm{L}$, $7.5 \times 10^{7}$ spores $/ \mathrm{mL}$ ) was dropped onto the coleoptiles of 6-day-old seedlings. To promote infection, the coleoptiles were wounded with a scalpel. Scale bar, $1 \mathrm{~cm}$. f Statistics for lesion length on the F. graminearum-infected coleoptiles ( $6 \mathrm{dpi}$ ) of Ubil::AtALA1 transgenic lines and wild-type maize. Six-day-old seedlings were treated with F. graminearum $\left(7.5 \times 10^{7} \mathrm{spores} / \mathrm{mL}\right)$. Data are presented as box-and-whisker plots of three replicates (eight plants each). $g$ Rot symptoms of ears ( $40 \mathrm{dpi}$ ) of Ubi7::AtALA1 transgenic lines and wild-type maize. Maize ears (three days after pollination) of Ubi7::AtALA1 transgenic lines and wild-type were inoculated with F. graminearum $\left(5 \times 10^{5} \mathrm{spores} / \mathrm{mL}\right)$. Mock, maize ears (three days after pollination) of Ubi7::AtALA1 transgenic lines and wild-type plants were inoculated with deionized water as a control. Scale bar, $2 \mathrm{~cm}$. $\mathrm{h}$ Statistics for the incidence rate of $F$. graminearum-infected maize ears. Data are presented as box-and-whisker plots of three replicates (five maize ears each). i Statistics for the diseased area ratio of $F$. graminearuminfected maize ears, shown as dot plots of three replicates (four ears each from individual plants). $\mathbf{j}$ DON concentration in the seeds from F. graminearuminfected corncobs of Ubi1::AtALA1 transgenic lines and wild-type maize. DON concentration was determined by LC-MS/MS. The results are shown as dot plots of three replicates (each replicate had $80 \mathrm{~g}$ seeds from four ears, which were harvested from individual plants). Box-and-whisker plots show the medians (horizontal lines), upper and lower quartiles (box edges), and 1.5× the interquartile range (whiskers). Different letters in $\mathbf{b}, \mathbf{f}$, and $\mathbf{h}-\mathbf{j}$ represent significant differences at $P<0.05$ by one-way ANOVA with Tukey multiple comparisons test. n.s., not significant. 


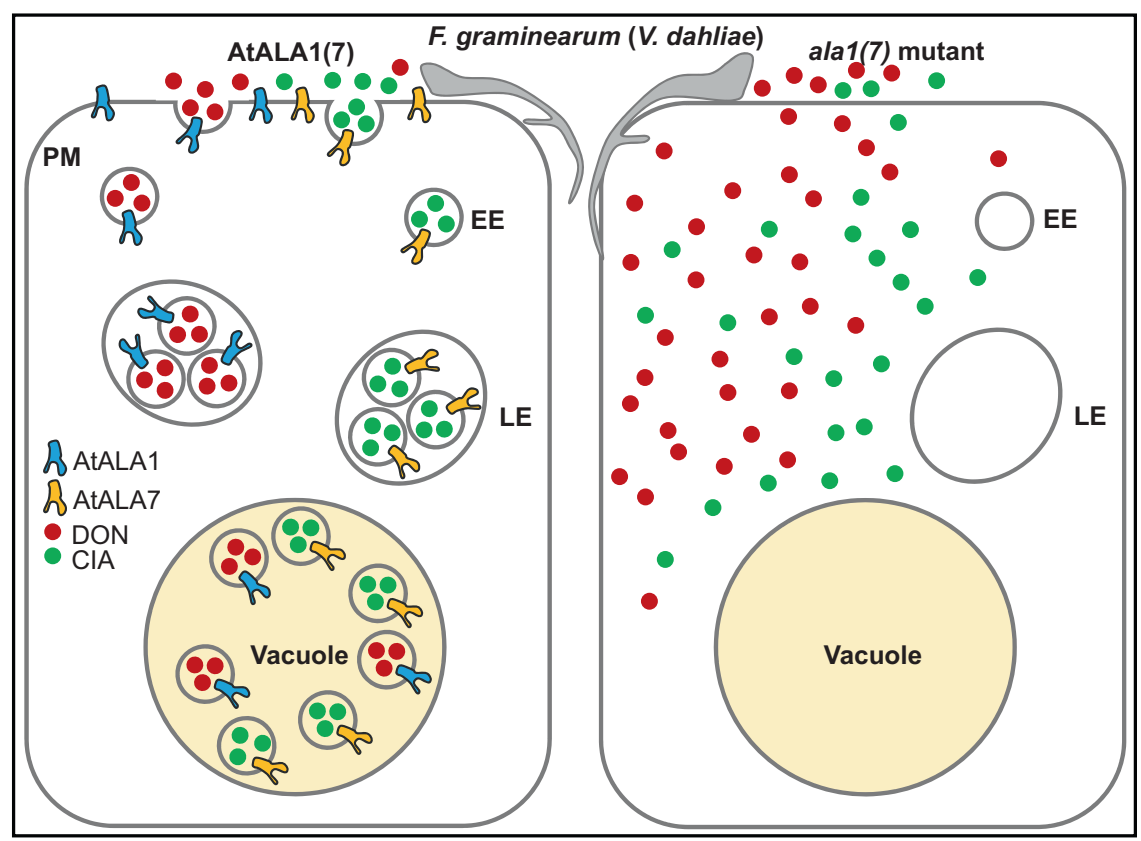

Fig. 7 A model showing Arabidopsis AtALA1-/AtALA7-mediated vesicle trafficking of DON/CIA for mycotoxin detoxification. Toxins (DON or CIA) are wrapped into vesicles on the PM, and the formation of these structures is mediated by P4 ATPases (AtALA1/AtALA7). Then, the toxins are transported into vacuoles, where they are compartmented and degraded. Lipophilic CIA is capable of crossing the plasma membrane barrier into cells and produces wilt symptoms. With an unknown membrane-associated passive transporter or endocytosis/pinocytosis mechanism ${ }^{99}$, DON can enter the cell, resulting in cytotoxicity due to its interactions with a number of targets ${ }^{100-102}$. By avoiding contact with the targets, the vesicle/vacuolar compartmentation of the toxins protects the plant innate immune system from damage, thus allowing plants to maintain their innate immunity against the invasion of pathogens and consequently increasing resistance to mycotoxin-associated diseases, such as Fusarium head blight (FHB) and Verticillium wilt. PM plasma membrane; EE early endosome, LE late endosome; DON deoxynivalenol, CIA cinnamyl acetate.

The DI was calculated according to the following formula: DI $=\left[\sum\right.$ (disease grades $\times$ number of infected plants)/ (total checked plants $\times 4)] \times 100$.

DON and CIA tolerance assays. For the DON and CIA treatments in Arabidopsis, 6-day-old plants were transferred to MS solid medium containing DON $(50 \mu \mathrm{g} / \mathrm{mL}$, APEXBIO) and CIA $(100 \mu \mathrm{g} / \mathrm{mL}$, Sigma-Aldrich) for $7 \mathrm{~d}$ or $9 \mathrm{~d}$, respectively. For DON treatment in maize, germinated seeds with radicle lengths of $\sim 0.5 \mathrm{~cm}$ were transferred to sterile water containing DON $(60 \mu \mathrm{M})$ for $2 \mathrm{~d}$.

Quantification of DON concentration by LC-MS/MS. Eighty grams of maize seeds and two grams of Arabidopsis siliques infected with F. graminearum were used for DON content analysis. The frozen tissue samples were ground to a fine powder in the presence of liquid nitrogen. Four volumes of methanol-water (80:20, $\mathrm{v} / \mathrm{v}$ ) were added to the powder, and the mixture was then shaken for $3 \mathrm{~h}$ at room temperature. After centrifugation at $5000 \mathrm{~g}$ for $10 \mathrm{~min}$, the supernatant was transferred to an Eppendorf tube and concentrated to dry by vacuum. The concentrate was resuspended in methanol-water $(50: 50, \mathrm{v} / \mathrm{v})$ and filtered through a $0.22 \mu \mathrm{m}$ pore filter membrane. Forty microlitres of the dissolved samples were used for DON quantification using a 4000 Q-Trap LC-ESI-MS/MS system (SCIEX, USA) with a C18 column $(2.1 \times 150 \mathrm{~mm}, 3.5 \mu \mathrm{m}$, Agilent $)$ at $30^{\circ} \mathrm{C}^{111}$. A series of gradients of methanol $/ 0.1 \%$ formic acid $(\mathrm{v} / \mathrm{v})$ was applied at a flow rate of $0.165 \mathrm{~mL} / \mathrm{min}$ as follows: $0 \sim 10 \mathrm{~min}, 22 \%$ to $40 \% ; 10 \sim 15 \mathrm{~min}, 40-100 \%$; 15 25 min, $100 \% ; 25 \sim 40 \mathrm{~min}, 22 \%$. DON was detected in the positive ion mode. Each test was repeated with three biological replicates.

Inhibitor treatments and FM4-64 staining. DON was labeled with 5-carboxyfluorescein (5-FAM, HY-66022, APEXBIO). CIA was labeled with fluorescein-isothiocyanate (FITC, HY-66019, MCE). The labeled compounds were produced by Fanbo Biochemicals Co. Ltd. (Haidian HighTech Business Park, Beijing, China).

For the CIA ${ }^{\text {FITC }}$ endocytosis inhibition assay, wild-type Arabidopsis seedlings were incubated in MS medium (Murashige and Skoog medium, M519, Phytotech) containing CIA ${ }^{\mathrm{FITC}}(5 \mu \mathrm{g} / \mathrm{mL}), \mathrm{FM} 4-64(8 \mu \mathrm{M}, \mathrm{F} 34653$, Thermo Scientific), and extra TyrA23 (50 $\mu \mathrm{M}$, APEXBIO), BFA ( $50 \mu \mathrm{M}$, Klamar) or Wm ( $33 \mu \mathrm{M}$, Solarbio) for $6 \mathrm{~h}$. For the DON ${ }^{5-F A M}$ endocytosis inhibition assay, wild-type Arabidopsis seedlings were treated with DON 5 -FAM $(9 \mu \mathrm{g} / \mathrm{mL})$ and FM4-64 $(8 \mu \mathrm{M})$ for $12 \mathrm{~h}$ (Mock), or mock with TyrA23 $(50 \mu \mathrm{M})$, BFA $(50 \mu \mathrm{M})$ or Wm $(33 \mu \mathrm{M})$. The samples were washed with deionized water three times before imaging.
Scanning electron microscopy (SEM). For the observation of mycelium and conidia of F. graminearum on the surface of Arabidopsis buds, the buds were frozen in liquid nitrogen, transferred into the cryostorage of a preparation chamber for platinum coating, and then imaged using an S-3400N SEM (Hitachi) at an accelerating voltage of $2 \mathrm{kV}$.

Living cell imaging. Living cell images were mainly acquired with a Leica SP8 pointscanning confocal system (Leica Microsystems, Germany). Images of the root meristem zone were acquired with a $\times 40$ objective, and root hair cells were acquired with a $\times 63$ objective. The pinhole aperture was 1 . EGFP, CIA ${ }^{\mathrm{FITC}}$, and DON ${ }^{5-\mathrm{FAM}}$ (green channel) were excited at $488 \mathrm{~nm}$, and the emission spectra were between 495 and $540 \mathrm{~nm}$. FM4-64 and RFP (red channel) were excited at $552 \mathrm{~nm}$, and the emission spectra were between 570 and $610 \mathrm{~nm}$. The scan speed was $400 \mathrm{~Hz}$ at a resolution of $1024 \times 1024$ pixels. All fluorescent signals were quantified using ImageJ.

For the observation of $F$. graminearum mycelium on Arabidopsis siliques, the SZX-ILLB2-200 stereomicroscope imaging system (Olympus) was used.

Statistical analysis. Statistical analyses were performed with Student's $t$ test or one-way ANOVA with a Tukey multiple comparisons test. Each experiment was repeated at least three times. The fluorescence intensity, $\mathrm{Rp}$, and Rs were calculated using the image processing program ImageJ (http://rsbweb.nih.gov/ij/). Standard errors and standard deviations were calculated using Excel (Microsoft, version 16) and SPSS (IBM, version 19).

Reporting summary. Further information on research design is available in the Nature Research Reporting Summary linked to this article.

\section{Data availability}

The LC-MS/MS data have been deposited in Metabolights with the data set identifier MTBLS3522. Data supporting the major findings of this work are available within the paper and its supplementary files. All additional data are available from the corresponding author upon request. Source data are provided with this paper.

Received: 7 January 2021; Accepted: 18 October 2021; Published online: 05 November 2021 


\section{References}

1. Talbot, N. J. On the trail of a cereal killer: exploring the biology of Magnaporthe grisea. Annu. Rev. Microbiol. 57, 177-202 (2003).

2. Howlett, B. J. Secondary metabolite toxins and nutrition of plant pathogenic fungi. Curr. Opin. Plant Biol. 9, 371-375 (2006).

3. Nesic, K., Ivanovic, S. \& Nesic, V. Fusarial toxins: secondary metabolites of Fusarium fungi. Rev. Environ. Contam. Toxicol. 228, 101-120 (2014).

4. Desjardins, A. E. et al. Reduced virulence of trichothecene-nonproducing mutants of Gibberella zeae in wheat field tests. Microbiology 9, 775-781 (1996).

5. Goswami, R. S. \& Kistler, H. C. Heading for disaster: Fusarium graminearum on cereal crops. Mol. Plant Pathol. 5, 515-525 (2004).

6. Matthew, N. \& McMullen. Affective contrast and assimilation in counter factual thinking. J. Exp. Soc. Psychol. 33, 77-100 (1997).

7. Trail, F. For blighted waves of grain: Fusarium graminearum in the postgenomics era. Plant Physiol. 149, 103-110 (2009).

8. Wang, H. et al. Horizontal gene transfer of Fhb7 from fungus underlies Fusarium head blight resistance in wheat. Science 368, eaba5435 (2020).

9. Logrieco, A., Moretti, A., Perrone, G. \& Mulè, G. Biodiversity of complexes of mycotoxigenic fungal species associated with Fusarium ear rot of maize and Aspergillus rot of grape. Int. J. Food Microbiol. 119, 11-16 (2007).

10. Logrieco, A., Bottalico, A., Mulé, G., Moretti, A. \& Perrone, G. Epidemiology of mycotoxin producing fungi. 645-667 (Springer, 2003).

11. Ma, Z. et al. Germplasms, genetics and genomics for better control of disastrous wheat Fusarium head blight. Theor. Appl. Genet. 133, 1541-1568 (2020).

12. Alexander, N. J., McCormick, S. P., Waalwijk, C., van der Lee, T. \& Proctor, R. $\mathrm{H}$. The genetic basis for 3-ADON and 15-ADON trichothecene chemotypes in Fusarium. Fungal Genet. Biol. 48, 485-495 (2011).

13. Bai, G.-H., Desjardins, A. \& Plattner, R. Deoxynivalenol-nonproducing Fusarium graminearum causes initial infection, but does not cause Disease Spread in wheat spikes. Mycopathologia 153, 91-98 (2002).

14. Jansen, C. et al. Infection patterns in barley and wheat spikes inoculated with wild-type and trichodiene synthase gene disrupted Fusarium graminearum. Proc. Natl. Acad. Sci. 102, 16892-16897 (2005).

15. Masuda, D. et al. Phytotoxic effects of trichothecenes on the growth and morphology of Arabidopsis thaliana. J. Exp. Bot. 58, 1617-1626 (2007).

16. McCormick, S. P., Stanley, A. M., Stover, N. A. \& Alexander, N. J. Trichothecenes: from simple to complex mycotoxins. Toxins 3, 802-814 (2011).

17. Pestka, J. J. Mechanisms of deoxynivalenol induced gene expression and apoptosis. Food Addit. Contam. Part A Chem. Anal. Control Expo. Risk Assess. 25, 1128-1140 (2008).

18. Berthiller, F. et al. Masked mycotoxins: a review. Mol. Nutr. Food Res. 57, 165-186 (2013).

19. D'Mello, J. P. F., Placinta, C. M. \& Macdonald, A. M. C. Fusarium mycotoxins: a review of global implications for animal health, welfare and productivity. Anim. Feed Sci. Technol. 80, 183-205 (1999).

20. Hathout, A. S. \& Aly, S. E. Biological detoxification of mycotoxins: a review. Ann. Microbiol. 64, 905-919 (2014).

21. Bhat, R. G. \& Subbarao, K. V. Host range specificity in Verticillium dahliae. Phytopathology 89, 1218-1225 (1999).

22. Baeza-Montañez, L., Gómez-Cabrera, R. \& García-Pedrajas, M. First report of Verticillium wilt caused by Verticillium dahliae on mango trees (Mangifera indica) in southern Spain. Plant Dis. 94, 380-380 (2010).

23. Christen, A. \& Peaden, R. Verticillium wilt in alfalfa. Plant Dis. 65, 319-321 (1981).

24. Fradin, E. F. \& Thomma, B. P. Physiology and molecular aspects of Verticillium wilt diseases caused by $V$. dahliae and V. albo-atrum. Mol. Plant Pathol. 7, 71-86 (2006).

25. Jiménez-Díaz, R. M. et al. Verticillium Wilt, a major threat to olive production: current status and future prospects for its management. Plant Dis. 96, 304-329 (2012)

26. Keykhasaber, M., Thomma, B. P. \& Hiemstra, J. A. Verticillium wilt caused by Verticillium dahliae in woody plants with emphasis on olive and shade trees. Eur. J. Plant Pathol. 150, 21-37 (2018).

27. Klosterman, S. J., Atallah, Z. K., Vallad, G. E. \& Subbarao, K. V. Diversity, pathogenicity, and management of Verticillium species. Annu. Rev. Phytopathol. 47, 39-62 (2009).

28. Koike, S. T., Subbarao, K. V., Davis, R. M., Gordon, T. R. \& Hubbard, J. C. Verticillium wilt of cauliflower in California. Plant Dis. 78, 1116-1121 (1994).

29. Larkin, R. P., Honeycutt, C. W. \& Olanya, O. M. Management of Verticillium wilt of potato with disease-suppressive green manures and as affected by previous cropping history. Plant Dis. 95, 568-576 (2011).

30. Pegg, G. F. \& Brady, B. L. Verticillium wilts. (CABI, 2002).

31. Subbarao, K. V., Kabir, Z., Martin, F. \& Koike, S. Management of soilborne diseases in strawberry using vegetable rotations. Plant Dis. 91, 964-972 (2007).
32. Li, G. et al. Mutation of a histidine-rich calcium-binding-protein gene in wheat confers resistance to Fusarium head blight. Nat. Genet. 51, 1106-1112 (2019).

33. Ferrigo, D., Raiola, A. \& Causin, R. Fusarium toxins in cereals: occurrence, legislation, factors promoting the appearance and their management. Molecules 21, 627 (2016).

34. Wang, Y. et al. Significant improvement of cotton Verticillium wilt resistance by manipulating the expression of Gastrodia antifungal proteins. Mol. Plant 9 1436-1439 (2016)

35. Gunupuru, L., Perochon, A. \& Doohan, F. Deoxynivalenol resistance as a component of FHB resistance. Trop. Plant. Pathol. 42, 175-183 (2017).

36. Poppenberger, B. et al. Detoxification of the Fusarium mycotoxin deoxynivalenol by a UDP-glucosyltransferase from Arabidopsis thaliana. J. Biol. Chem. 278, 47905-47914 (2003).

37. Li, X. et al. Transgenic wheat expressing a barley UDP-glucosyltransferase detoxifies deoxynivalenol and provides high levels of resistance to Fusarium graminearum. Mol. Plant Microbe Interact. 28, 1237-1246 (2015).

38. Bernardo, A. N., Ma, H., Zhang, D. \& Bai, G. Single nucleotide polymorphism in wheat chromosome region harboring Fhb1 for Fusarium head blight resistance. Mol. Breed. 29, 477-488 (2012)

39. Pumphrey, M. O., Bernardo, R. \& Anderson, J. A. Validating the Fhb1 QTL for Fusarium head blight resistance in near-isogenic wheat lines developed from breeding populations. Crop Sci. 47, 200-206 (2007).

40. Su, Z. et al. A deletion mutation in TaHRC confers Fhb1 resistance to Fusarium head blight in wheat. Nat. Genet. 51, 1099-1105 (2019).

41. Husar, S. et al. Overexpression of the UGT73C6 alters brassinosteroid glucoside formation in Arabidopsis thaliana. BMC Plant Biol. 11, 1-14 (2011)

42. Berthiller, F. et al. Occurrence of deoxynivalenol and its 3- $\beta$-D-glucoside in wheat and maize. Food Addict. Contam. 26, 507-511 (2009).

43. Chain, E.P.o.C.i.t.F. Scientific opinion on the risks for human and animal health related to the presence of modified forms of certain mycotoxins in food and feed. EFSA J. 12, 3916 (2014).

44. Nachmias, A., Buchner, V. \& Krikun, J. Comparison of proteinlipopolysaccharide complexes produced by pathogenic and non-pathogenic strains of Verticillium dahliae Kleb. from potato. Physiol. Plant Pathol. 20, 213-221 (1982).

45. Meyer, R., Slater, V. \& Dubery, I. A. A phytotoxic protein-lipopolysaccharide complex produced by Verticillium dahliae. Phytochemistry 35, 1449-1453 (1994).

46. Jia, Z.-Q., Shi, F.-M., Yuan, H.-Y., Hou, Y.-Y. \& Li, Y.-Z. Comparison of methods of purification and identification of Verticillium dahliae toxins. J. Life Sci. 1, 67-73 (2007).

47. Nachmias, A., Buchner, V. \& Burstein, Y. Biological and immunochemical characterization of a low molecular weight phytotoxin isolated from a protein -lipopolysaccharide complex produced by a potato isolate of Verticillium dahliae Kleb. Physiol. Plant Pathol. 26, 43-55 (1985).

48. Daly, J. M. \& Deverall, B. J. Toxins and plant pathogenesis. (Academic Press, 1983).

49. Mansoori, B., Milton, J. \& Smith, C. Isolation and partial purification of a phytotoxin related to pathogenic Verticillium species. J. Phytopathol. 143, 33-36 (1995).

50. Laouane, H., Sedra, M. H. \& Lazrek, H. B. Isolation and characterization of a new phytotoxic molecule from culture fluids of Verticillium dahliae. Global J. Sci. Front. Res. Chemistry 13, 7-B (2013).

51. D'Orazio, V. et al. Phytotoxic metabolites produced by Verticillium dahliae Kleb in olive wilting: a chemical and spectroscopic approach for their molecular characterisation. Nat. Prod. Res. 35, 1991-2001 (2019).

52. Baldridge, R. D. \& Graham, T. R. Two-gate mechanism for phospholipid selection and transport by type IV P-type ATPases. Proc. Natl. Acad. Sci. 110, E358-E367 (2013)

53. Graham, T. R. Flippases and vesicle-mediated protein transport. Trends Cell Biol. 14, 670-677 (2004).

54. Pomorski, T. G. \& Menon, A. K. Lipid somersaults: uncovering the mechanisms of protein-mediated lipid flipping. Prog. Lipid Res. 64, 69-84 (2016).

55. Roland, B. P. \& Graham, T. R. Directed evolution of a sphingomyelin flippase reveals mechanism of substrate backbone discrimination by a P4-ATPase. Proc. Natl. Acad. Sci. 113, E4460-E4466 (2016).

56. Vestergaard, A. L. et al. Critical roles of isoleucine-364 and adjacent residues in a hydrophobic gate control of phospholipid transport by the mammalian P4-ATPase ATP8A2. Proc. Natl. Acad. Sci. 111, E1334-E1343 (2014).

57. Shin, H. W. \& Takatsu, H. Substrates of P4-ATPases: beyond aminophospholipids (phosphatidylserine and phosphatidylethanolamine). FASEB J. 33, 3087-3096 (2019)

58. Best, J. T., Xu, P. \& Graham, T. R. Phospholipid flippases in membrane remodeling and transport carrier biogenesis. Curr. Opin. Cell Biol. 59, 8-15 (2019). 
59. Nintemann, S. J., Palmgren, M. \& Lopez-Marques, R. L. Catch you on the flip side: a critical review of flippase mutant phenotypes. Trends Plant Sci. 24, 468-478 (2019).

60. Andersen, J. P. et al. P4-ATPases as phospholipid flippases-structure, function, and enigmas. Front. Physiol. 7, 275 (2016).

61. López-Marqués, R. L., Davis, J. A., Harper, J. F. \& Palmgren, M. Dynamic membranes: the multiple roles of P4 and P5 ATPases. Plant Physiol. 185, 619-631 (2020).

62. López-Marqués, R. L., Gourdon, P., Günther Pomorski, T. \& Palmgren, M. The transport mechanism of P4 ATPase lipid flippases. Biochem. J. 477, 3769-3790 (2020).

63. Lopez-Marques, R. L., Theorin, L., Palmgren, M. G. \& Pomorski, T. G. P4ATPases: lipid flippases in cell membranes. Pflügers Arch. Eur. J. Physiol. 466, 1227-1240 (2014).

64. McMahon, H. T. \& Gallop, J. L. Membrane curvature and mechanisms of dynamic cell membrane remodelling. Nature 438, 590-596 (2005).

65. Rothman, J. E. \& Wieland, F. T. Protein sorting by transport vesicles. Science 272, 227-234 (1996).

66. Van der Mark, V. A., Elferink, R. P. \& Paulusma, C. C. P4 ATPases: flippases in health and disease. Int. J. Mol. Sci. 14, 7897-7922 (2013).

67. Gomès, E., Jakobsen, M. K., Axelsen, K. B., Geisler, M. \& Palmgren, M. G. Chilling tolerance in Arabidopsis involves ALA1, a member of a new family of putative aminophospholipid translocases. Plant Cell 12, 2441-2454 (2000).

68. McDowell, S. C., Lopez-Marques, R. L., Poulsen, L. R., Palmgren, M. G. \& Harper, J. F. Loss of the Arabidopsis thaliana P4-ATPase ALA3 reduces adaptability to temperature stresses and impairs vegetative, pollen, and ovule development. PLoS ONE 8, e62577 (2013).

69. Niu, Y. et al. ALA6, a P4-type ATPase, is involved in heat stress responses in Arabidopsis thaliana. Front. Plant Sci. 8, 1732 (2017).

70. Zhu, B. et al. Arabidopsis ALA1 and ALA2 mediate RNAi-based antiviral immunity. Front. Plant Sci. 8, 422 (2017).

71. Underwood, W., Ryan, A. \& Somerville, S. C. An Arabidopsis lipid flippase is required for timely recruitment of defenses to the host-pathogen interface at the plant cell surface. Mol. Plant 10, 805-820 (2017).

72. Guo, Z. et al. Lipid flippases promote antiviral silencing and the biogenesis of viral and host siRNAs in Arabidopsis. Proc. Natl. Acad. Sci. USA 114, 1377-1382 (2017).

73. Zhang, X. et al. Arabidopsis flippases cooperate with ARF GTPase exchange factors to regulate the trafficking and polarity of PIN auxin transporters. Plant Cell 32, 1644-1664 (2020)

74. Poulsen, L. R. et al. The Arabidopsis P4-ATPase ALA3 localizes to the Golgi and requires a $\beta$-subunit to function in lipid translocation and secretory vesicle formation. Plant Cell 20, 658-676 (2008).

75. Davis, J. A. et al. The lipid flippases ALA4 and ALA5 play critical roles in cell expansion and plant growth. Plant Physiol. 182, 2111-2125 (2020).

76. McDowell, S. C., López-Marqués, R. L., Poulsen, L. R., Palmgren, M. G. \& Harper, J. F. Harper loss of the Arabidopsis thaliana P4-ATPase ALA3 reduces adaptability to temperature stresses and impairs vegetative, pollen, and ovule development. PLoS One 8, e62577 (2013)

77. McDowell, S. C. et al. Loss of the Arabidopsis thaliana P4-ATPases ALA6 and ALA7 impairs pollen fitness and alters the pollen tube plasma membrane. Front. Plant Sci. 6, 197 (2015).

78. Zhou, Y. et al. The tip-localized phosphatidylserine established by Arabidopsis ALA3 is crucial for rab gtpase-mediated vesicle trafficking and pollen tube growth. Plant Cell 32, 3170-3187 (2020).

79. Sanz-Fernández, M. et al. Screening Arabidopsis mutants in genes useful for phytoremediation. J. Hazard. Mater. 335, 143-151 (2017).

80. Ovecka, M. et al. Structural sterols are involved in both the initiation and tip growth of root hairs in Arabidopsis thaliana. Plant Cell 22, 2999-3019 (2010).

81. Saito, C. et al. A complex and mobile structure forms a distinct subregion within the continuous vacuolar membrane in young cotyledons of Arabidopsis. Plant J. 29, 245-255 (2002).

82. López-Marqués, R. L., Poulsen, L. R. \& Palmgren, M. G. A putative plant aminophospholipid flippase, the Arabidopsis P4 ATPase ALA1, localizes to the plasma membrane following association with a $\beta$-subunit. PLoS One 7, e33042 (2012)

83. Boutté, Y. et al. Endocytosis restricts Arabidopsis KNOLLE syntaxin to the cell division plane during late cytokinesis. EMBO J. 29, 546-558 (2010).

84. Banbury, D. N., Oakley, J. D., Sessions, R. B. \& Banting, G. Tyrphostin A23 inhibits internalization of the transferrin receptor by perturbing the interaction between tyrosine motifs and the medium chain subunit of the AP2 adaptor complex. J. Biol. Chem. 278, 12022-12028 (2003).

85. Dhonukshe, P. et al. Clathrin-mediated constitutive endocytosis of PIN auxin efflux carriers in Arabidopsis. Curr. Biol. 17, 520-527 (2007)

86. Nebenführ, A., Ritzenthaler, C. \& Robinson, D. G. Brefeldin A: deciphering an enigmatic inhibitor of secretion. Plant Physiol. 130, 1102-1108 (2002).

87. Kong, S.-G. et al. Blue light-induced association of phototropin 2 with the Golgi apparatus. Plant J. 45, 994-1005 (2006).
88. Schu, P. V. et al. Phosphatidylinositol 3-kinase encoded by yeast VPS34 gene essential for protein sorting. Science 260, 88-91 (1993).

89. Wurmser, A. E., Gary, J. D. \& Emr, S. D. Phosphoinositide 3-kinases and their FYVE domain-containing effectors as regulators of vacuolar/lysosomal membrane trafficking pathways. J. Biol. Chem. 274, 9129-9132 (1999).

90. Urban, M., Daniels, S., Mott, E. \& Hammond-Kosack, K. Arabidopsis is susceptible to the cereal ear blight fungal pathogens Fusarium graminearum and Fusarium culmorum. Plant J. 32, 961-973 (2002).

91. Kemen, E. \& Jones, J. D. G. Obligate biotroph parasitism: can we link genomes to lifestyles? Trends Plant Sci. 17, 448-457 (2012).

92. Vleeshouwers, V. G. A. A. \& Oliver, R. P. Effectors as tools in disease resistance breeding against biotrophic, hemibiotrophic, and necrotrophic plant pathogens. Mol. Plant Microbe Interact 27, 196-206 (2014).

93. Diamond, M. et al. The fusarium mycotoxin deoxynivalenol can inhibit plant apoptosis-like programmed cell death. PLoS One 8, e69542 (2013).

94. Desmond, O. J. et al. The Fusarium mycotoxin deoxynivalenol elicits hydrogen peroxide production, programmed cell death and defence responses in wheat. Mol. Plant Pathol. 9, 435-445 (2008).

95. Tian, J. et al. Expression of baculovirus anti-apoptotic genes p35 and op-iap in cotton (Gossypium hirsutum L.) enhances tolerance to Verticillium wilt. PLoS One 5, e14218 (2010).

96. Lemmens, M. et al. The ability to detoxify the mycotoxin deoxynivalenol colocalizes with a major quantitative trait locus for Fusarium head blight resistance in wheat. Mol. Plant Microbe Interact. 18, 1318-1324 (2005).

97. Bowles, D., Lim, E.-K., Poppenberger, B. \& Vaistij, F. E. Glycosyltransferases of lipophilic small molecules. Annu. Rev. Plant Biol. 57, 567-597 (2006).

98. Laouane, H. \& Lazrek, H. Synthesis and toxicity evaluation of cinnamyl acetate: a new phytotoxin produced by a strain of Verticillium dahliae pathogenic on olive tree. Int. J. Agric. Biol. 13, 444-446 (2011).

99. Maresca, M. From the gut to the brain: Journey and pathophysiological effects of the food-associated trichothecene mycotoxin deoxynivalenol. Toxins $\mathbf{5}$, 784-820 (2013).

100. McLaughlin, C. et al. Inhibition of protein synthesis by trichothecenes. Mycotoxins in human and animal health (1977).

101. Rocha, O., Ansari, K. \& Doohan, F. Effects of trichothecene mycotoxins on eukaryotic cells: a review. Food Addit. Contam. 22, 369-378 (2005).

102. McLean, M. The phytotoxicity ofFusarium metabolites: An update since 1989 Mycopathologia 133, 163-179 (1996).

103. Zheng, X. et al. The cauliflower mosaic virus (CaMV) 35 S promoter sequence alters the level and patterns of activity of adjacent tissue- and organ-specific gene promoters. Plant Cell Rep. 26, 1195-1203 (2007).

104. Armstrong, C., Green, C. \& Phillips, R. Development and availability of germplasm with high Type II culture formation response. Maize genetics cooperation news letter (USA) (1991).

105. Zhang, M. et al. Spatiotemporal manipulation of auxin biosynthesis in cotton ovule epidermal cells enhances fiber yield and quality. Nat. Biotechnol. 29, 453 (2011).

106. Frame, B. R. et al. Production of transgenic maize from bombarded type II callus: effect of gold particle size and callus morphology on transformation efficiency. In Vitro Cell. Dev. Biol. Plant 36, 21-29 (2000).

107. Clough, S. J. \& Bent, A. F. Floral dip: a simplified method for Agrobacteriummediated transformation of Arabidopsis thaliana. Plant J. 16, 735-743 (1998)

108. Cuomo, C. A. et al. The Fusarium graminearum genome reveals a link between localized polymorphism and pathogen specialization. Science 317, 1400-1402 (2007).

109. Zhang, X.-W. et al. In planta stage-specific fungal gene profiling elucidates the molecular strategies of Fusarium graminearum growing inside wheat coleoptiles. Plant Cell 24, 5159-5176 (2012).

110. Xu, L. et al. Functional characterization of cotton genes responsive to Verticillium dahliae through bioinformatics and reverse genetics strategies. $J$. Exp. Bot. 65, 6679-6692 (2014).

111. Zeng, J. et al. Cytokinin inhibits cotton fiber initiation by disrupting PIN3amediated asymmetric accumulation of auxin in the ovule epidermis. J. Exp. Bot. 70, 3139-3151 (2019).

\section{Acknowledgements}

This work was supported by the Chinese Ministry of Science and Technology of China (Grant 2016YFD0100505 to Y.P.), National Transgenic New Species Breeding Major Project of China (2016ZX08005-003-004 to Y.P.), Chongqing Research Program of Basic Research and Frontier Technology (cstc2017jcyjB0316 to X.L.), and the Graduate Student Research Innovation Project of Chongqing (CYB17072 to F.W.). We thank Dr. Caixia Gao (Institute of Genetics and Developmental Biology, Chinese Academy of Sciences) for her help in maize transformation, professor Zhiying Ma (Hebei Agricultural University, China), and professor Guiliang Jian (The Institute of Plant Protection, Chinese Academy of Agricultura Sciences) for their kind donation of $V$. dahliae strain L2-1 and V991. We are grateful to professor Zhengqiang Ma (Nanjing Agricultural University, Nanjing, China) and Dr. Dapeng Zhang (USDA ARS, BARC, PSI) for their critical reading of the manuscript. 


\section{Author contributions}

Y.P. conceived and supervised the project. F.W. performed ALAs' identification and subcellular localization, plant transformation, assays of disease resistance and toxin resistance, plant management, and data analysis; Y.L. and H.R. participated in P4 ATPases identification and protein subcellular localization; X.L. J.H., M.S., J.Z., Y.C., H.L., J.Z., and S.S. participated in plant transformation and plant management; L.H., J.Z, Y.F., X.Y., D.J., and M.Z. participated in toxin determination, confocal and SEM observations, and data processing. Y.P. and F.W. wrote the paper with input from all other authors.

\section{Competing interests}

The authors declare no competing interests.

\section{Additional information}

Supplementary information The online version contains supplementary material available at https://doi.org/10.1038/s41467-021-26727-5.

Correspondence and requests for materials should be addressed to Yan Pei.

Peer review information Nature Communications thanks Rong Di and the other, anonymous, reviewer(s) for their contribution to the peer review of this work. Peer reviewer reports are available.
Reprints and permission information is available at http://www.nature.com/reprints

Publisher's note Springer Nature remains neutral with regard to jurisdictional claims in published maps and institutional affiliations.

\section{(c) (i)}

Open Access This article is licensed under a Creative Commons Attribution 4.0 International License, which permits use, sharing, adaptation, distribution and reproduction in any medium or format, as long as you give appropriate credit to the original author(s) and the source, provide a link to the Creative Commons license, and indicate if changes were made. The images or other third party material in this article are included in the article's Creative Commons license, unless indicated otherwise in a credit line to the material. If material is not included in the article's Creative Commons license and your intended use is not permitted by statutory regulation or exceeds the permitted use, you will need to obtain permission directly from the copyright holder. To view a copy of this license, visit http://creativecommons.org/ licenses/by/4.0/.

(C) The Author(s) 2021 